\title{
Thymol-based Chitosan Nanogels Have Strong Antibacterial and Anti-biofilm Effects on Multidrug- resistant Pathogens
}

\section{Tohid Piri Gharaghie ( $\nabla$ tohidpirie@yahoo.com )}

Department of Biotechnology, Faculty of Basic Sciences, Islamic Azad University, Shahrekord Branch, Shahrekord, Iran https://orcid.org/0000-0002-0041-5842

\section{Sheida Biranvand}

Islamic Azad University shahrekord branch

\section{Neda Jegargoshe Shirin}

islamic azad university damghan branch

\section{Yalda Elahianfar}

Iran University of Medical Sciences

\section{Somayeh Ghahari}

Sari Agricultural Sciences and Natural Resources University

\section{Sajjad Ghahari}

Shahid Chamran University of Ahvaz

\section{Research}

Keywords: MDR, Chitosan, Antibiofilm, Thymol, Antibacterial

Posted Date: December 18th, 2020

DOl: https://doi.org/10.21203/rs.3.rs-128664/v1

License: (9) This work is licensed under a Creative Commons Attribution 4.0 International License.

Read Full License 


\title{
Thymol-based chitosan nanogels have strong antibacterial and anti-biofilm effects on multidrug-resistant pathogens
}

\author{
Tohid Piri Gharaghie ${ }^{1, *}$, Sheida Biranvand ${ }^{2}$, Neda Jegargoshe Shirin ${ }^{3}$, Yalda Elahianfar ${ }^{4}$, Somayeh \\ Ghahari $^{5}$, Sajjad Ghahari ${ }^{6}$
}

\begin{abstract}
${ }^{1}$ Department of Biotechnology, Faculty of Basic Sciences, Islamic Azad University, Shahrekord Branch, Shahrekord, Iran ${ }^{2}$ Department of Biotechnology, Faculty of Basic Sciences, Islamic Azad University, Shahrekord Branch, Shahrekord, Iran ${ }^{3}$ Department of Biotechnology, Faculty of Basic Sciences, Islamic Azad University, Damghan Branch, Semnan, Iran ${ }^{4}$ Department of Medical Education, School of Medicine, Iran University of Medical Sciences , Tehran, Iran..

${ }^{5}$ Sari University of Agricultural Sciences and Natural Resources, Genetics and Agricultural Biotechnology Institute of Taberestan (GABIT), Sari, Iran

${ }^{6}$ Department of Biology, Faculty of Basic Sciences, Shahid Chamran University, Ahvaz Branch, Ahvaz, Iran.

* Corresponding Author: Associate Professor, Department of Biology, Shahrekord Branch, Islamic Azad University, Shahrekord, Iran.

Email Author Interface: tohidpirie@yahoo.com
\end{abstract}

Abstract
Introduction: 
The discovery of antibiotics for the treatment of bacterial infections was one of the most important advances in medical history, but unfortunately bacteria are highly adaptable, and overuse of antibiotics has made many bacteria resistant to antibiotics. In the present study, the antibacterial and antibiofilm effects of Thymol-based chitosan nanogels were investigated.

\section{Materials \& Methods:}

After clinical screening of MDR strains from the hospital environment, the morphological characteristics of the synthesized nanoparticles were identified using SEM, DLS, XRD and FTIR and the efficiency of encapsulation, stability and drug release were evaluated. After determining the MIC concentration of nanoparticles, the expression of OmpA and PgaB biofilm genes were determined by q-Real-Time PCR and the antibacterial and cytotoxic properties of the nanoparticles were determined by well diffusion and MTT methods, respectively.

\section{Results:}

Three bacteria Pseudomonas, Acinetobacter and Staphylococcus were identified as MDR strains and the antibacterial and antibiofilm properties of nanoparticles with a size of $82.71 \mathrm{~nm}$, encapsulation efficiency of $76.54 \%$ and stability up to 60 days at $4{ }^{\circ} \mathrm{C}$ were evaluated. The results of the biological study showed strong antibacterial properties of Thymol-based chitosan nanoparticles by reducing the expression of OmpA and PgaB biofilm genes at a significant level of $\mathrm{P} \leq 0.05$ and reducing antibiotic resistance compared to the free drug thymol and chitosan nanogels. Thymol-based chitosan nanogels at concentrations of 0.125 to $256 \mu \mathrm{g} / \mathrm{mL}$ showed the lowest cytotoxicity against HEK-293 compared to chitosan and free Thymol nanogels.

\section{Discussion and Conclusion:}

The results of the study showed the very strong antibacterial properties of Thymol-based chitosan nanogels against MDR strains such as Staphylococcus, Acinetobacter and Pseudomonas as the challenging bacteria of the century. Therefore, the use of Thymol-based chitosan nanogels can be reported as a new application strategy with high potential in the pharmaceutical industry.

Keywords: MDR, Chitosan, Antibiofilm, Thymol, Antibacterial

\section{Introduction}


A nosocomial infection is an infection that the patient does not have at the time of hospitalization and during the recovery period. The prevalence of nosocomial infections is high, so that their risk also is high in the best-equipped hospitals [1]. According to studies, for every 100 patients admitted to different wards of hospitals in developed and developing countries, $7 \%$ and $10 \%$ of them get nosocomial infections, respectively [2]. The increasing prevalence of nosocomial infections has led to significant problems, including; more extended hospital stays, microbial resistance, and even mortality rates [3]. The significant rate of nosocomial infections is related to the development of respiratory diseases [4]; however, comprehensive studies have not been conducted on the classification of nosocomial infections, the maximum range of damage of these infections, the density of diseases created, and the thickness of bacteria involved. On the other hand, the prevalence of drug resistance following nosocomial infections limits treatment options, lowers the effectiveness of antibiotics, and, eventually, the beginning of the "post-antibacterial" period [5]. Besides, the indiscriminate use (overuse) of antibiotics, by killing weaker bacteria, artificially reconstructed the natural selection process and leading to the selection of more potent bacteria as the selected survivors [6]. This process after this referred to as secondary selection. Identification of resistant bacteria screened in the natural selection process, their density in different hospital environments, Rate of occurrence of various diseases, and determining their MDR pattern is significant [7]. Antibiotic-resistant microorganisms after the secondary selection process can create resistance to other drug compounds by various methods such as biofilm formation and transmits this property to future generations [8]. Due to the multifactorial nature of biofilm formation in bacteria, several genes and their products involved in this process, including genes expressing $O m p A$ [9], pgaB [10], and so on. These proteins play a vital role in adhesion and biofilm formation in bacteria. OmpA is a purine and one of the major outer membrane proteins with a molecular weight of $38 \mathrm{kDa}$ that is effective in creating biofilms, interacting with human epithelial cells, and their relationship with each other [9]. $P G A_{A B C D}$, on the other hand, is an operon in bacteria involved in the production of poly- $\beta(1,6)-N$-acetyl-D-glucosamine (PNAG). PNAG affects biofilm development by binding to abiotic surfaces and intercellular adhesion. All $P G A$ genes required for optimal biofilm formation under different growth conditions [10]. $\mathrm{PgaB}$ is a two-barrel domains periplasmic protein with an $\mathrm{N}$-terminal family of four carbohydrate esterases (CE4) that exhibits metal-dependent acetylation activity on PNAG oligomers and enhances its efficiency [11]. Today, failure in treating infectious diseases has become commonplace, and multi-antibiotic-resistant strains are on the rise [12]. Due to the lack of new antimicrobial compounds to treat MDR (multidrug resistance) bacterial infections and the slowness of making new effective antibiotics, increasing attention should be paid to natural compounds as adjunctive therapies [13]. Medicinal plants, due to their small compositions, considered as critical new sources of treatment for various diseases, including infectious diseases [14]. Thymol is one of the small useful compounds of thyme (Thymus vulgaris L., Lamiaceae) plant whose antimicrobial effects have proven at every stage of the growth cycle $[15,16]$. Despite all the antimicrobial properties, limitations such as; Low solubility in water, ease of degradation, chemical reaction, and chemical and biological instability due to volatility have limited the use of Thymol as an effective antimicrobial and preservative compound [17]. Nanobiotechnology, with improving biocompatibility, uses various biomaterials to make nanostructures such as nanoparticles [18]. Nanoparticles, due to their small size, increase the stability of the substance in the bloodstream and can carry higher amounts of the drugs due to their unique physical and chemical properties [19]. Recently, natural chitosan polymer with low toxicity and biodegradable and biocompatible properties is used in 
the manufacture of pharmaceutical nanocarriers [20]. Chitosan is a carrier of a biodegradable drug in the body with the ability to adhere to mucosal cells, which with its permeability can help open strong epithelial connections. Also, chitosan has considered as a biopolymer that has antimicrobial activity in a wide variety of fungi and bacteria [21]. Because Thymol is sensitive to light, the formulation of this compound with chitosan as a carrier could lead to a new drug formulation against virulence and biofilm of Dangerous pathogens.

\section{Materials and methods}

\subsection{Clinical studies}

\subsubsection{Ethics and Patients}

During June to October 2019, from the patients referred to the Infectious Diseases Clinic of Rasoul Akram Hospital in Tehran, A total of 200 patients who complained of symptoms such as fever and chills, dizziness and drowsiness, burning when urinating, the strange smell of urine, respiratory problems, muscle and chest pain, cough with yellow, green or bloody mucus were selected and informed consent and ethical approval was received from these patients. The initial diagnosis of the disease in these people was made by the fellow doctor. This study was conducted in compliance with the ethical principles and regulations approved by Rasoul Akram Hospital, Iran.

\subsubsection{Diagnosis of disease}

According to the fellow doctor, the history of smoking, weakened immune system and history of chronic disease was recorded in individuals. For screening, blood, urine, respiratory samples (bronchial lavage, secretions suction), and chest $\mathrm{x}$-ray were performed according to the fellow doctor's instructions.

\subsubsection{Prescribing antibiotics and clinical examination of patients}

After diagnosing the infection, the decision about the type of antibiotic to be used to treat the infection was made separately by the fellow doctor based on each individual's symptoms. Prescribed antibiotics include; Ciprofloxacin, erythromycin, chloramphenicol, amikacin, tetracycline, imipenem, tazobactam, ampicillin-sulbactam, kanamycin, ceftazidime, and clistin. After 15 days, the results of controlling or not controlling the disease were recorded by a fellow doctor. A sampling of patients was also performed.

\subsection{Laboratory detection}

\subsubsection{Recovery of bacterial isolates and biochemical tests}

From patients who did not recover after antibiotic administration, blood, urine, and respiratory samples were collected in the hospital laboratory, and biochemical tests, including growth on blood agar, McCangi agar, and oxidase, catalase, urease, lysine-decarboxylase, MR-VP, SIM, TSA, Simon citrate and arginine tests were performed.

\subsubsection{Detection of resistant bacteria}


Initial screening was performed by a physician to diagnose resistant bacteria. Also, sampling was performed on patients who did not recover after antibiotic treatment. After the initial diagnosis of bacteria, an antibiotic susceptibility test was performed by standard disk diffusion method according to CLSI (Clinical and Laboratory Standard Institute) protocol and antibiotic discs of imipenem (IMI) $(10 \mu \mathrm{g})$, ciprofloxacin (CIP) $(5 \mu \mathrm{g})$, erythromycin (E) (15 $\mu \mathrm{g}$ ), chloramphenicol (C) (30 $\mu \mathrm{g})$, amikacin (AK) $(10 \mu \mathrm{g})$, tetracycline (T) $(30 \mu \mathrm{g})$, clindamycin $(2 \mu \mathrm{g})$, kanamycin (K) (30 $\mu \mathrm{g})$, ceftazidime (CAZ) (30 $\mu \mathrm{g})$ and cleistine (CO) $(5 \mu \mathrm{g})$ (MAST, England). Pseudomonas ATCC 27853, Acinetobacter ATCC 17978, and Staphylococcus ATCC BAA-2856 were used as positive controls.

\subsubsection{Detection of biofilm-forming bacteria}

\subsubsection{Biofilm detection phenotypic test}

According to the method of Mariana et al. [22], Congo Red Agar (CRA) medium was prepared by combining BHI (brain heart infusion broth) $(37 \mathrm{~g} / \mathrm{L})$, sucrose $(50 \mathrm{~g} / \mathrm{L})$, agar $(10 \mathrm{~g} / \mathrm{L})$ and Congo Red indicator $(0.8 \mathrm{~g} / \mathrm{L})$ and clinical strains of bacteria were cultured on plates containing Congo Red culture medium for 24 hours at $37^{\circ} \mathrm{C}$. Under such conditions, biofilm-producing bacteria form black colonies and other bacteria form red colonies.

\subsubsection{Biofilm detection genotype test}

In this test, after culturing the bacteria, $200 \mu \mathrm{L}$ of them was added to 96 -well sterile polystyrene wells and incubated for 24 hours at $37{ }^{\circ} \mathrm{C} .200 \mu \mathrm{L}$ of $1 \%$ violet crystal dye was added to it for staining for 15 minutes and then washed with PBS. After adding $200 \mu \mathrm{L}$ of 33\% acetic acid, absorbance was measured at $570 \mathrm{~nm}$ by ELISA Reader Stat Fax2100 (Awareness Technology, Ukraine). In order to investigate by Optical density cut-off (ODc) method, first the standard deviation and mean OD of negative control wells and ODc were calculated according to Formula 1 and OD of the studied wells was classified according to Table 1.

Formula 1: ODc $=$ Average OD of negative control wells $+(3 \times$ Standard deviation of negative control wells $)$

Table 1. Classification of bacteria based on biofilm formation capacity

\begin{tabular}{|c|c|}
\hline Biofilm Class & Results \\
\hline OD $>4 \times$ ODc & Strong biofilm \\
\hline $2 \times$ ODc $<$ OD $\leq 4 \times$ ODc & Medium biofilm \\
\hline ODc $<$ OD $\leq 2 \times$ ODc & Poor biofilm \\
\hline OD $\leq$ ODc & Negative biofilm \\
\hline
\end{tabular}

\subsubsection{Molecular detection of bacteria and confirmation of the presence of biofilm genes}


Initially, DNA extraction from screened resistant bacteria was performed according to the protocol of the DNA extraction kit (Sinaclon, Iran). Also, to detect bacterial densities in clinical specimens, polymerase chain reaction (PCR) method using universal primer and sequence (F: 5'-AGAGTTTGATYMTGGCTCAG-3') and (R: 5'AGAAAGGAGGTGATCCAGCC-3') used. After determining the microbial density by polymerase chain reaction, the presence of Acinetobacter, Pseudomonas and Staphylococcus as well as the presence of OmpA and PgaB genes were confirmed. The primer sequences used in this study are listed in the Table 2.

Table 2: Sequence of primers used for molecular detection

\begin{tabular}{|c|c|c|c|}
\hline & 16SrRNA & OmpA & $P g a B$ \\
\hline Acinetobacter & $\begin{array}{l}\text { (F:5'-TAYCGYAAAGAYTTGAAAGAAG-3') } \\
\text { (R:5'-CMACACCYTTGTTMCCRTGA-3') }\end{array}$ & $\begin{array}{l}\text { (F:5'-CGCTTCTGCTGGTGCTGAAT-3') } \\
\text { (R:5'- CGTGCAGTAGCGTTAGGGTA-3') }\end{array}$ & $\begin{array}{l}\text { (F:5'-AAGAAAATGCCTGTGCCGACCA-3') } \\
\text { (R:5'- GCGAGACCTGCAAAGGGCTGAT-3') }\end{array}$ \\
\hline Pseudomonas & $\begin{array}{c}\text { (F:5'-CGAGGAGTAGTTTTCCCATGTTCACCT-3') } \\
\text { (R:5'- CCCGTAGAAATGCCTCGACTTC-3') }\end{array}$ & $\begin{array}{l}\text { (F:5'AGCGTTCGTCCTGCACAAGT-3') } \\
\text { (R:5'-TCCACCATGCTCAGGGAGAT-3') }\end{array}$ & $\begin{array}{l}\text { (F:5'- GCGTGCTCAAGTGTTCAAGG-3') } \\
\text { (R 5'- ATTCGCCAGCAACCGAAAAC-3') }\end{array}$ \\
\hline Staphylococcus & $\begin{array}{c}\text { (F:5' - ATCGTTTTATCGGGACCATC -3') } \\
\text { (R:5'- TCATTAACTACAACGTAATCGTA-3') }\end{array}$ & $\begin{array}{l}\text { (F:5'TATCTGGTGGCGTAACACCTG-3') } \\
\text { (R:5'-GATGAAGCCGTTACGTTGTTC-3') }\end{array}$ & $\begin{array}{l}\text { (F:5' - AGAACCTACAACTTCAGAACCTGTG-3') } \\
\text { (R 5'- AA ACCTTTAACCGTAGTTGGCGGTAT-3') }\end{array}$ \\
\hline
\end{tabular}

The final volume of the reaction $(25 \mu \mathrm{L})$ includes: $13 \mu \mathrm{L}$ PCR master mix (PCR buffer, $\mathrm{MgCl}_{2}, \mathrm{dNTP}, 0.2$ units of Taq polymerase), $1 \mu \mathrm{L}$ forward and reverse primer, $1 \mu \mathrm{L}$ template cDNA and $9 \mu \mathrm{L}$ distilled water (Amplicon, Denmark). The PCR cycle program containing $95^{\circ} \mathrm{C}$ at $5 \mathrm{~min}, 94^{\circ} \mathrm{C}$ at $1 \mathrm{~min}, 60^{\circ} \mathrm{C}$ at $1 \mathrm{~min}$, and $72{ }^{\circ} \mathrm{C}$ at $1 \mathrm{~min}(35$ cycles) and final expansion at $72{ }^{\circ} \mathrm{C}$ for $5 \mathrm{~min}$.

\subsection{Synthesis, encapsulation and characterization of nanoparticles}

\subsubsection{Preparation of chitosan nanogels}

1 gram of chitosan powder (Sigma-Aldrich, USA) was added to a container containing $1 \mathrm{~mL}$ of distilled water and 1 $\mathrm{mL}$ of hydrogen chloride and mixed for 1 hour using a magnetic stirrer with $800 \mathrm{rpm}$. It was then centrifuged at 1000 rpm for 5 minutes. Dissolved $50 \mathrm{mg}$ of myristic acid and $100 \mathrm{mg}$ of EDC and NHS in $1 \mathrm{ml}$ of ethanol and then the resulting solution was added dropwise to chitosan for 2 hours. The $\mathrm{pH}$ of the reaction was then increased with dilute sodium $(0.1 \mathrm{M})$ to precipitate chitosan nanogels. inally, the chitosan nanogel precipitate was separated by centrifugation and washed with ethanol and then distilled water.

\subsubsection{Thymol encapsulation in chitosan nanogels}

By adding dilute hydrochloric acid, the chitosan nanogel was dissolved and the resulting mixture was sonicated for 30 minutes using an ultrasonic homogenizer. $0.5 \mathrm{~mL}$ of $99.9 \%$ Thymol essential oil (Plant Therapy, USA) was dissolved in $2 \mathrm{~mL}$ of ethanol, and myristate was added dropwise at the same time as chitosan nanogel sonication. The sonication was continued for 15 minutes to encapsulate Thymol essential oil in nanogels.

\subsubsection{Characterization and investigation of drug loading in nanoparticles with FESEM, XRD, and FTIR electron microscopy}


Determination of particle size and the charge was performed using Dynamic light scattering devices and palladium electrodes of ZetaPals device (Brookhaven Instruments Corp., USA) at a light scattering angle of $90^{\circ}$ and at a temperature of $25^{\circ} \mathrm{C}$. The morphology of nanoparticles was investigated using field scanning electron microscopy (FESEM) model MIRA3 (TESCAN, Czech Republic) and XRD model X' Pert Pro (Panalytical, Netherlands). $20 \mu \mathrm{L}$ of dilute nanoparticle solution was dried on aluminum foil at room temperature and then coated with a layer of gold to conduct electrical conductivity. For FTIR spectroscopy, one percent of each sample was converted to a tablet by potassium bromide and then spectroscopy was performed in the frequency range of $400-4000 \mathrm{~cm}^{-1}$.

\subsubsection{Evaluation of encapsulation efficiency and release of encapsulated thymol in chitosan}

After the encapsulation of thymol in chitosan nanogels, encapsulation efficiency (EE) was evaluated. Then $1 \mathrm{~mL}$ of thymol-based chitosan was centrifuged for 1 hour at $14000 \mathrm{~g}$ at $4{ }^{\circ} \mathrm{C}$ and then washed with PBS. After determining the amount of encapsulated thymol in each sample by measuring the maximum adsorption of the supernatant at a wavelength of $653 \mathrm{~nm}$, the percentage of encapsulation efficiency was calculated using Formula 2.

Formula 2: EE\%= Total amount of initial drug entrapped into the Thymol formulations - amount of free drug in supernatant/ total amount of drug $\times 100$

The process of examining drug release in vitro was performed in a $12 \mathrm{kDa}$ dialysis bag (MWCO). For this purpose, after adding $2 \mathrm{~mL}$ of free thymol and $2 \mathrm{~mL}$ of thymol encapsulated in chitosan nanogels to the dialysis bag, the whole fraction was placed in $50 \mathrm{~mL}$ of PBS solution at different pHs of 3, 5, and 7.4 and gently mixed at $37{ }^{\circ} \mathrm{C}$ and $50 \mathrm{rpm}$. It was then aliquoted at specified intervals and a new environment was added to it. Different kinetic diffusion models were analyzed to evaluate drug release characteristics.

\subsection{Investigation of anti-biofilm properties of synthesized nanoparticles}

2.4.1. Determination of minimum inhibitory concentration (MIC) and minimum bactericidal concentration (MBC)

To determine the minimum inhibitory concentration (MIC) and minimum bactericidal concentration (MBC), the serial dilution method with three replications in a culture medium was used. Dilutions ranging from 256 to $1 \mu \mathrm{L} / \mathrm{mL}$ of chitosan nanoparticles, Thymol essential oil, and Thymol-based chitosan nanoparticles were added to the 96-well plate well. Then $100 \mu \mathrm{L}$ of microbial suspension was added to each well and the plates were incubated overnight at $37{ }^{\circ} \mathrm{C}$. One well was selected as blank (empty culture medium), and one well was selected as the positive control (culture medium + standard bacteria). Finally, the turbidity of all wells was measured using the ELISA Reader Stat Fax2100 (Awareness Technology, Ukraine) apparatus at $630 \mathrm{~nm}$. The values of MIC, MBC (the lowest concentration of drugs that inhibit bacterial growth or death, respectively), and subMIC (the highest concentrations of drugs that have no effect on bacterial growth) were determined.

\subsubsection{Evaluation of OmpA, and PgaB biofilm gene expression}


Genomic RNA was extracted according to the protocol of the RNX-Plus kit (Sinagen, Iran) from bacteria isolated from clinical specimens after treatment with subMIC concentrations of chitosan nanoparticles, Thymol essential oil, and Thymol-based chitosan nanoparticles. Concentrations of 1 to $2 \mu \mathrm{g}$ of RNA were determined by the Nanodrop Model One C (Thermo, USA) with a light absorption reading at $260 \mathrm{~nm}$. Also, light absorption was measured at 260/230 nm to ensure the absence of contamination and the light absorption ratio for RNA was considered equal to 2 . CDNA was constructed from RNA extracted from treated and untreated bacteria according to the YTA Kit Protocol (Yekta Tajhiz, Iran). Quantitative Real-Time PCR test was performed with Mastermix Cybergreen YTA (Yekta Tajhiz, Iran) and 16SrRNA gene as a reference gene. $15 \mu \mathrm{L}$ of reaction volume consisted of $0.5 \mu \mathrm{L}$ of cDNA, $0.5 \mu \mathrm{L}$ of the forward primer, $0.5 \mu \mathrm{L}$ of back primer, $10 \mu \mathrm{L}$ of mastermix, and $3.5 \mu \mathrm{L}$ of double sterile distillation water. The temperature cycle program also included initial denaturation at $95{ }^{\circ} \mathrm{C}$ for $10 \mathrm{~min}$, followed by 40 cycles at $95{ }^{\circ} \mathrm{C}$ for $20 \mathrm{sec}$ and $60^{\circ} \mathrm{C}$ for $40 \mathrm{sec}$. The final step to draw the Melting Curve was selected from 55 to $95{ }^{\circ} \mathrm{C}$.

\subsection{Investigation of antibacterial properties of synthesized nanoparticles}

After preparing the Müller Hinton agar culture medium, using a sterile pipette No. 5, made $4 \mathrm{~mm}$ deep wells on a plate containing the culture medium and then the suspension of resistant bacteria was cultured on the culture medium surface with a sterile swab. $30 \mu \mathrm{L}$ of subMIC concentration of chitosan nanoparticles, Thymol essential oil and Thymol-based chitosan nanoparticles were poured into wells and incubated for 24 hours. DMSO and gentamicin were used as negative control and positive control, respectively, and the diameter of the growth inhibition zone was measured.

\subsection{Investigation of cytotoxicity of synthesized nanoparticles}

HEK 293 cells are widely used in biocellular research due to their reliable growth. Therefore, to evaluate the cytotoxicity and survival rate of synthesized nanoparticles, the MTT tetrazolium salt-based colorimetric method was used on HEK 293 cells. According to the instructions of the DMA500 kalazist kit, cells were cultured in 96-well plates with 2-5 $\times 104$ cells per $100 \mu \mathrm{L}$ of culture medium and incubated overnight in a $\mathrm{CO}_{2}$ incubator. Then the fresh culture medium was replaced and incubation was performed at $37{ }^{\circ} \mathrm{C}$ for 24 hours. Dilutions of 256 to $0.125 \mu \mathrm{L} / \mathrm{mL}$ of chitosan nanogels, thymol essential oil, and thymol-based chitosan nanogels were first dissolved in DMSO and then diluted in a culture medium and added to the cells. After incubation of $\mathrm{CO}_{2}$ at $37{ }^{\circ} \mathrm{C}$ for 48 hours, $20 \mathrm{~mL}$ of MTT solution ( $5 \mathrm{mg} / \mathrm{mL}$ in the colorless buffer PBS) (Kalazist, Iran) was added to the wells and after 4 hours of incubation, the contents of the wells were drained and $100 \mu \mathrm{L}$ of DMSO solution was added. The plates were placed on a shaker at $400 \mathrm{rpm}$ for 6 minutes to completely dissolve the formed formazan crystals in DMSO. The color intensity was then read by the ELISA Reader Stat Fax2100 (Awareness Technology, Ukraine) at $570 \mathrm{~nm}$. The cell- free culture medium was used as the blank of the ELISA reader apparatus and the culture medium with the cell without drug was used as the control of the living cell. The percentage of viable cells was obtained from Formula 3.

Formula 3: Cell survival rate $\%=\frac{\text { treated cells OD }}{\text { Control cells } O D} \times 100$

\subsection{Statistical analysis}


Statistical analysis in this study was calculated using SPSS software version 16 and the results were subjected to Oneway Analysis Of Variance (ANOVA). Also, the expression of target genes between the control and treated samples was calculated by Tukey's HSD post statistical method.

\section{Results}

\subsection{Results of clinical studies}

The clinical part of the study was a descriptive-analytical study. The study population included 200 patients admitted to different wards of Rasoul Akram Hospital in the period from June 2019 to October 2019. To prove the infection, a set of patient profiles includes; Fever, urine test, blood culture and other secretions and chest x-ray were used. The required information was obtained through a checklist by daily examination by the fellow doctor and recording patient characteristics in the file. Patients' clinical signs were regularly examined by the fellow doctor. During the present study, 200 patients from the Infectious Diseases Clinic of Rasoul Akram Hospital were studied, of which 110 were female (55\%) and 90 male (45\%). Patients ranging in age from 18 to 65 years were selected with a mean age of $17.63 \pm 44$ for female patients and $53 \pm 19.77$ for male patients. According to the results of the initial clinical screening by the fellow doctor, the initial diagnosis of bacterial infection in patients was reported. In terms of length of hospital stay, for various reasons in Rasoul Akram Hospital, 70 female patients (63.63\%) were hospitalized for more than 20 days and 40 (36.36\%) patients were hospitalized for less than 20 days. Also in the male group, 57 patients (63.33\%) were hospitalized for more than 20 days and 33 patients (36.66\%) were hospitalized for less than 20 days. According to the results, there was a significant relationship between the length of hospital stay and the incidence of nosocomial infections, so that in patients who were hospitalized for more than 20 days for various reasons, the rate of infections (density and severity of infection) was higher. After sampling and evaluation of clinical features, patients were divided into 4 groups; Urinary tract infection, Bloodstream (sepsis), surgical wound infection and pneumonia. Of the 200 patients studied, 82 case urinary tract infections, 26 case sepsis, 49 case surgical wound infections, and 43 case pneumonia was reported by the fellow doctor. The most common nosocomial infections in the present study were urinary tract infection (UTI) (41\%), wound infection (24.5\%), pneumonia (21.5\%), and blood infection or sepsis (13\%), respectively. Also, the most common microbial strains causing nosocomial infections in the initial diagnosis of this study were Escherichia coli, Enterobacter species, Staphylococcus aureus, Pseudomonas aeruginosa, and Acinetobacter baumannii. After receiving written consent, patients were treated with antibiotics at the discretion of the fellow doctor and according to the patient's condition. After 15 days of treatment of patients with antibiotics according to the fellow doctor's prescription, clinical signs were evaluated and the symptoms of urinary tract infection, sepsis, surgical wound infection, and pneumonia did not improve in 27, 19, 34, and 32 patients, respectively, which indicate a wide range of antibiotic resistance. According to the results of this study, the most common nosocomial infections after antibiotic therapy are; Wound infections (17\%), pneumonia (16\%), UTI (13.5\%), and sepsis (9.5\%). Examining the resistance of infections to antibiotic therapy, it was found that bacteria causing sepsis and pneumonia show more resistance to antibiotic therapy. Out of 26 patients with sepsis, only 7 (26.9\%) recovered after antibiotic therapy and $19(73.09 \%)$ did not recover, which indicates a significant difference in response to antibiotic treatment. Also, in pneumonia, out of 43 patients, only $25.58 \%$ (11 patients) recovered after antibiotic therapy; While the major 
rate of patients (79.06\%, 34 patients) did not respond to antibiotic therapy. Regarding urinary tract infection and wound infection, respectively; $67.07 \%$ (55 patients) and 30.61\% (15 patients) recovered, and $32.92 \%$ (27 patients) and $69.38 \%$ (34 patients) did not respond well to antibiotic therapy. Thus, the rates of resistant bacteria in pneumonia and sepsis were higher than wound infection and urinary tract infection, respectively, which indicates the failure of antibiotic treatment in these infections. These patients were selected as a source of MDR bacterial isolation and sampling was performed for laboratory evaluation.

\subsection{Laboratory diagnosis results}

Of the samples collected from 112 unhealed patients, after biochemical tests, the most common bacterial densities were related to gram-negative organisms and Escherichia coli, Acinetobacter, Pseudomonas aeruginosa, and other Enterobacter species. Then, antibiotic susceptibility testing was performed and the highest resistance to broadspectrum antibiotics was related to Acinetobacter, Staphylococcus, and Pseudomonas, respectively. Phenotypic biofilm detection test was performed in isolated strains according to Table 3.

Table 3: Screening of patients and clinical specimens for isolation of resistant bacteria

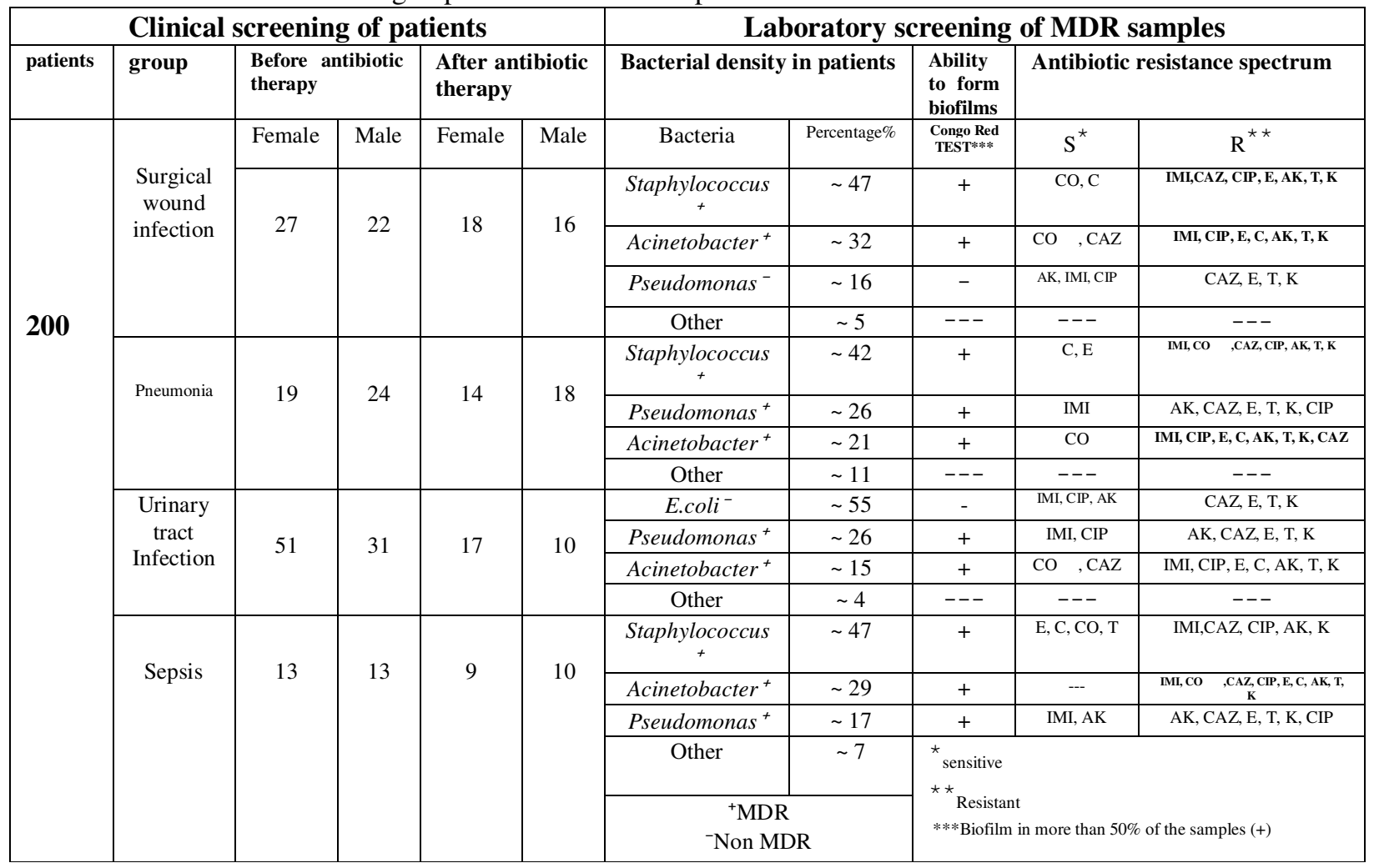

Resistant bacteria were then selected for isolation and species identification and PCR was performed with 16SrRNA primer and 27 pathogens with 110 bp length were identified as Ab, Pseudomonas aeruginosa, and Staphylococcus. According to the aim of the study to evaluate the strength of biofilm in isolated strains, a microtiter test was performed and the results showed strong and moderate biofilms in 18 and 9 samples of each bacterium, respectively (Graph 1). 


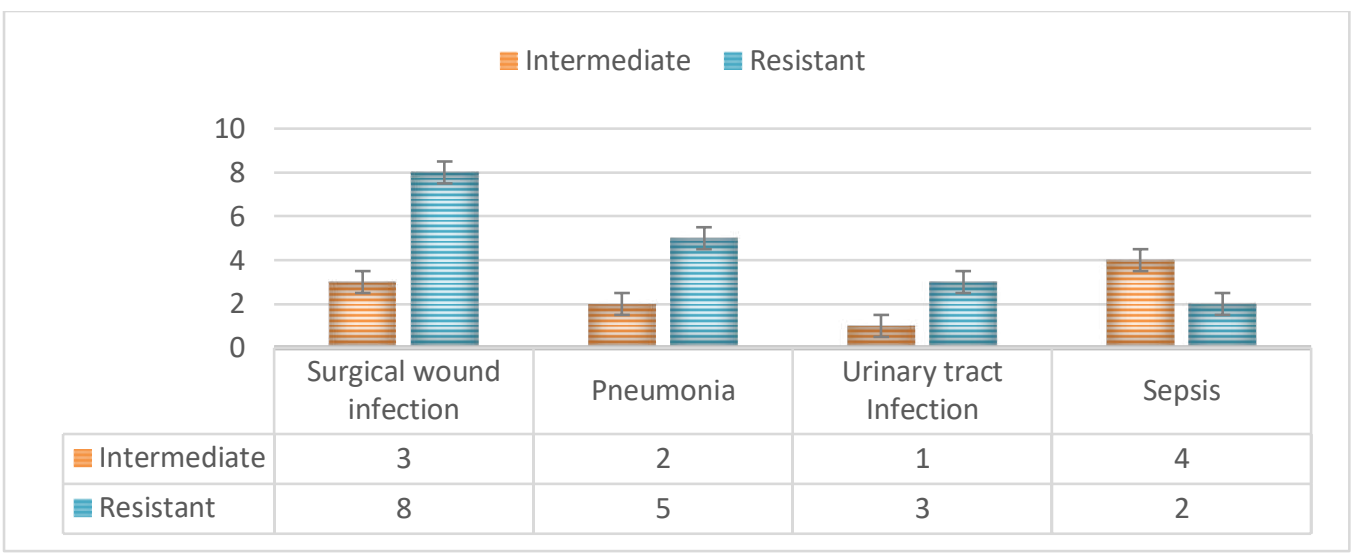

Graph 1. Capacity and strength of biofilm in screened strains, by standard plate microtiter method

To evaluate the presence of biofilm genes in bacteria, PCR test was performed using specific primers and confirmed the presence of $\operatorname{OmpA}$ and $p g a B$ genes in the screened bacteria. Standard strains were used as positive control in this test. Then, chitosan nanogels and Thymol-based chitosan nanoparticles were synthesized and optimized in terms of size, charge and shape. The average size of chitosan nanogels and Thymol-based chitosan nanoparticles were 72.35 and $82.71 \mathrm{~nm}$ with spherical structure, respectively (Figure 1).

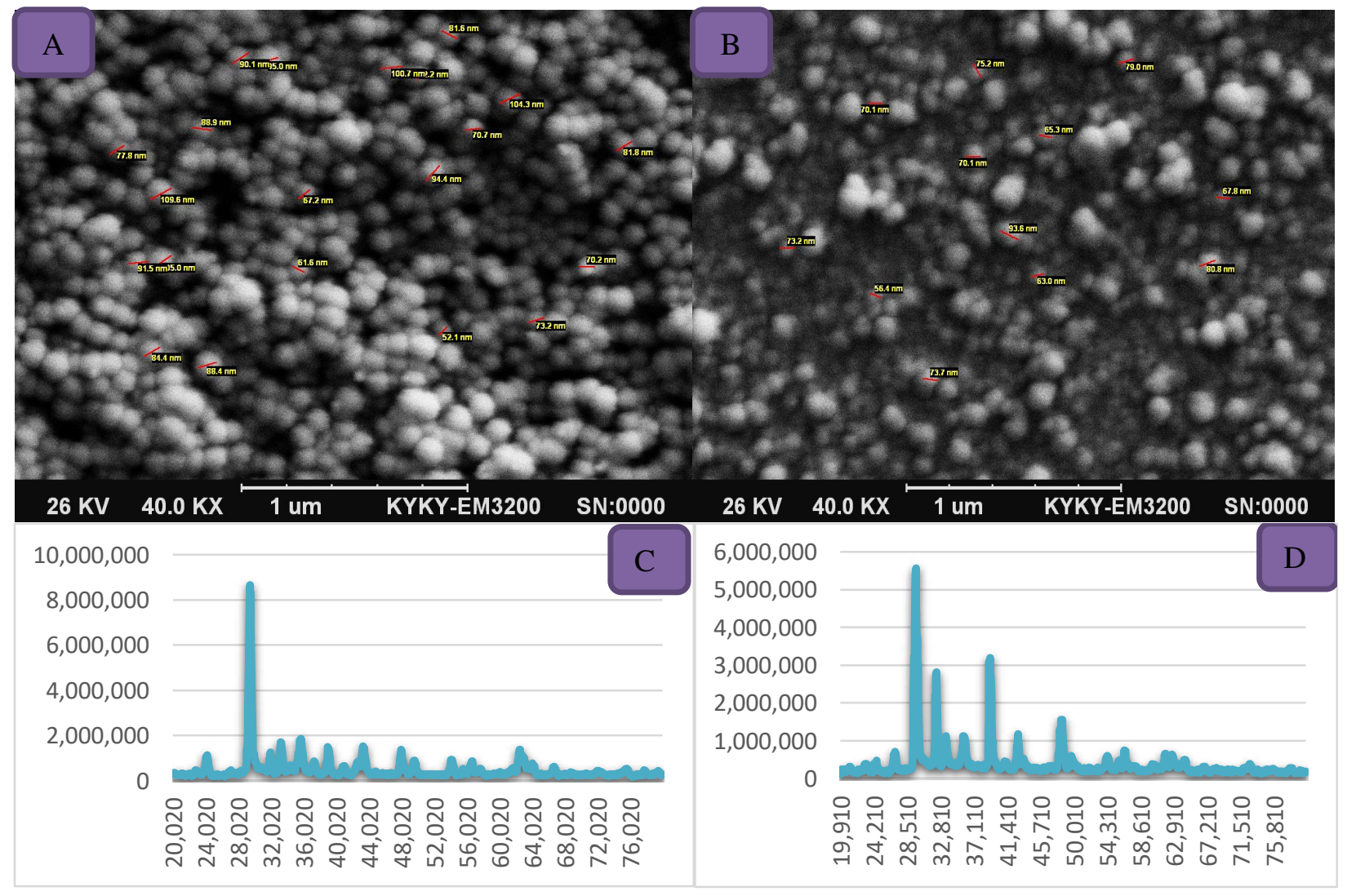

Figure 1. A) SEM image of chitosan; B) SEM image of chitosan nanoparticles containing Thymol; C) XRD diagram of chitosan nanoparticles; D) XRD diagram of chitosan nanoparticles containing Thymol 
Suitable uniformity of the produced nanoparticles was determined using the DLS (Dynamic Light Scattering) technique. The Poly Dispersity Index (PDI) and mean diameter of chitosan nanogels were 0.178 and $258.2 \mathrm{~nm}$, respectively, and for Thymol-based chitosan nanoparticles were 0.115 and $179.3 \mathrm{~nm}$, respectively. The zeta potential of drug-free chitosan nanogels and thymol-based chitosan showed 64.19 and $66.35 \mathrm{mV}$, respectively. Also according to the XRD diagram, in the range of $2 \theta$, peaks with higher intensities have more reflection, which indicates the presence of nanoparticles. Accordingly, the highest reflections related to peak intensities at 29.470 angles in Figure 2C and angles 32.470, and 38.940 in Figure 2D were related to chitosan nanogels and Thymol-based chitosan nanoparticles, respectively; Therefore, the amount of nanoparticles formed in the range of these peaks is higher. FTIR test was performed and in the spectrum of chitosan nanoparticles, peaks in the range of $2858.54 \mathrm{~cm}^{-1}$ and $2928.83 \mathrm{~cm}^{-}$ ${ }^{1}$ were related to $\mathrm{C}-\mathrm{H}$ bond and peak $3329.24 \mathrm{~cm}^{-1}$ belonged to the amino group of chitosan and peaks of 1082.15 , 1158.47 and $1739.11 \mathrm{~cm}^{-1}$ belonged to the carboxyl group. In the FTIR spectrum of the thymol, the peak of 2919.19 $\mathrm{cm}^{-1}$ is associated with the tensile vibration of $\mathrm{C}-\mathrm{H}$ in the benzene ring. The absorption peaks at 1742.34, 1630.90, 1460.77, and 1378.23 were four unequal stretches corresponded to a specific peak of Thymol. The peaks at 1247.83 and $1098.26 \mathrm{~cm}^{-1}$ were related to the $-\mathrm{OH}$ and $\mathrm{C}-\mathrm{O}$ flexural vibrations. The peak of $3370.00 \mathrm{~cm}^{-1}$ belongs to the O-H group. The presence of these peaks in Thymol-based chitosan nanoparticles indicates the loading of Thymol in the chitosan nanogel (Figure 2).
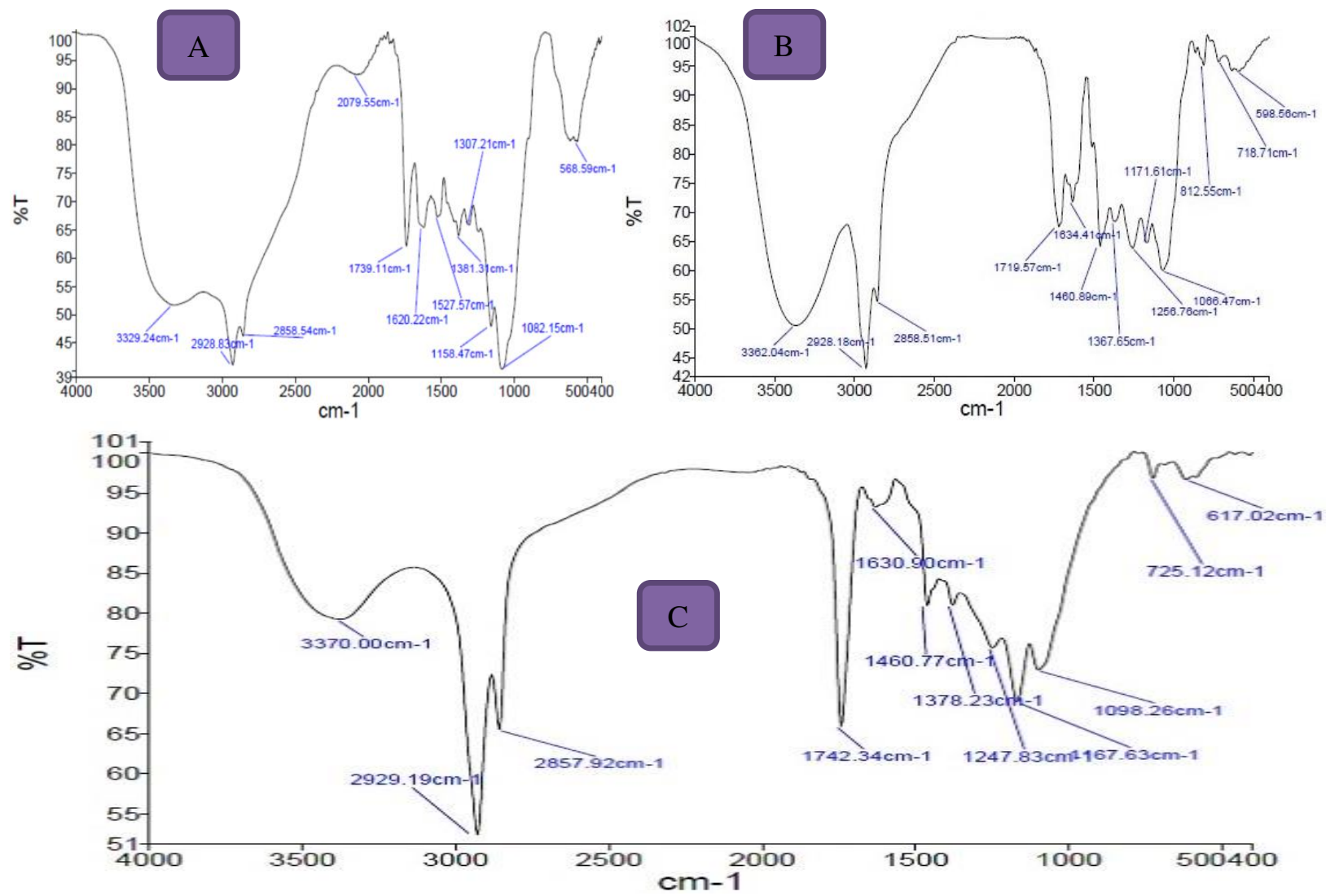
Figure 2. A) FTIR spectrum of chitosan; B) FTIR spectrum of Thymol free drug; c) FTIR spectrum of chitosan nanoparticles containing Thymol

According to Table 4, encapsulation efficiency (EE), PDI dispersion index, hydrodynamic diameter size and surface charge of nanoparticles indicate proper synthesis and high encapsulation efficiency of Thymol in chitosan nanogels.

Table 4. Morphological features of synthesized nanoparticles. Data are represented as mean $\pm \mathrm{SD}, \mathrm{n}=3$

\begin{tabular}{|l|l|l|l|l|l|}
\hline Nanomaterials & $\begin{array}{l}\text { Polydispersity index } \\
(\mathrm{PDI})\end{array}$ & Surface charge $(\mathrm{mV})$ & $\begin{array}{l}\text { Vesicle size } \\
(\mathrm{nm})(\mathrm{SEM})\end{array}$ & $\begin{array}{l}\text { Hydrodynamic } \\
\text { diameter }(\mathrm{nm})\end{array}$ & EE (\%) \\
\hline Chitosan nanogels & $0.178 \pm 0.024$ & $64.19 \pm 2.62$ & 72.35 & $258.2 \mathrm{~nm} \pm 6.38$ & --- \\
\hline $\begin{array}{l}\text { Thymol-based } \\
\text { chitosan nanogels }\end{array}$ & $0.115 \pm 0.005$ & $66.35 \pm 1.75$ & 82.71 & $179.3 \pm 5.26 \mathrm{~nm}$ & $76.54 \pm 0.62$ \\
\hline
\end{tabular}

A dialysis bag containing Thymol-free drug and Thymol-based chitosan nanogel was used to evaluate the controlled release profile of the drug. The resulting curve is similar to the bacterial growth curve in that in the first 9 hours; the Thymol-free drug is released rapidly, followed by a slow release of the drug from the dialysis bag. This reduction in emissions lasted up to 48 hours. According to the results, the release of Thymol-free drug and Thymol-based chitosan nanogel in the first 9 hours was $88 \%$ and $69 \%$, respectively, and then the drug release rate was proven so that after 48 hours, the release rate of the two drugs increased to 97 and $76.54 \%$, respectively. Morphological properties including; mean size, Poly Dispersity Index (PDI), and encapsulation efficiency (EE) were measured to evaluate the stability of Thymol-based nanogels at different temperatures and time intervals. The results showed that with increasing temperature, it changed the morphological properties of chitosan-based nanogels that as a result of EE drug reduction was reduced. However, increasing the shelf life of the drug has little effect on the morphological characteristics and EE of the drug (Figure 3).
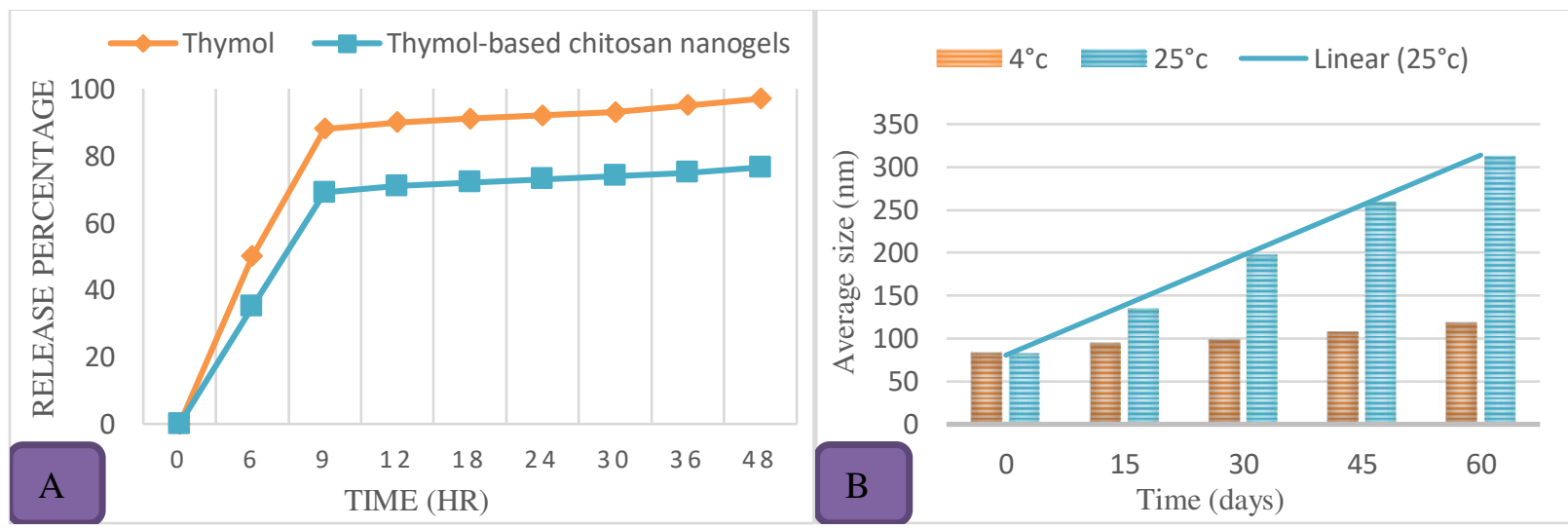


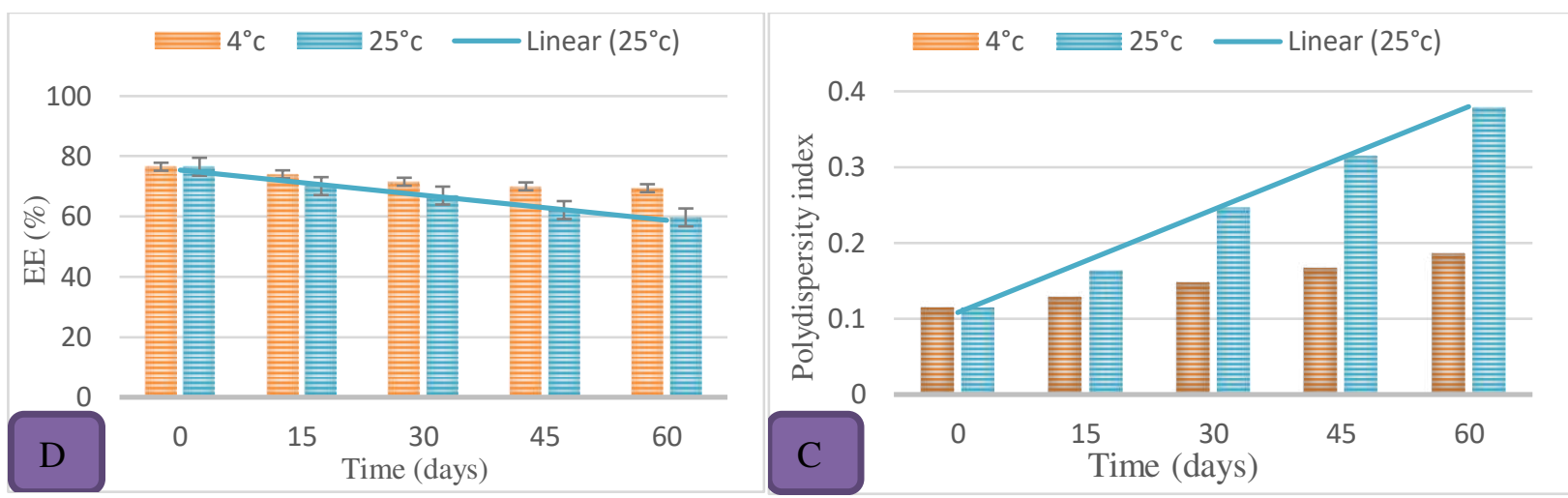

Figure 3. (A) Evaluation of drug release test; (B) the effect of time and storage temperature on mean size; (C) PDI index; (D) encapsulation efficiency of Thymol-based chitosan nanoparticles.

\subsection{Investigation of inhibitory ability of synthesized nanoparticles}

The susceptibility of 27 biofilm-producing isolates in 3 bacterial genera was determined based on the minimum growth inhibitory concentration (MIC) for chitosan nanogels, Thymol essential oil, and Thymol-based chitosan nanoparticles. Bacterial positive biofilm strains were exposed to $100 \%$ to $0.39 \%$ of essential oils and nanoparticles for 24 hours. According to Table 5, each strain had a range of MICs with concentrations between 256 and $1 \mu \mathrm{g} / \mathrm{mL}$. All isolates were treated with Sub-MIC concentrations of chitosan nanoparticles, Thymol essential oil, and Thymol-based chitosan nanoparticles. According to the results, Thymol-based chitosan nanoparticles had the highest inhibition and then chitosan nanoparticles and Thymol essential oil had the highest inhibition in all 3 bacterial genera, respectively. Pseudomonas was also less resistant than the other two genera. The highest resistance to synthesized compounds belonged to the genus Staphylococcus. After determining the MIC of nanoparticles in 3 bacterial genera, samples S4, S12, and S27 were identified as the most resistant pathogens, respectively, and were selected to evaluate the efficiency of the synthesized nanoparticles. For this purpose, fresh cultures of bacteria were first prepared and a colony of each bacterium was cultured in a tube containing the Müller-Hinton broth medium. Then S4, S12, and S27 bacteria were cultured at concentrations of 256 to $1 \mu \mathrm{g} / \mathrm{mL}$ chitosan nanogels and thymol-based chitosan nanoparticles and during 24 hours of incubation at $37^{\circ} \mathrm{C}$ every 4 hours light absorption of the samples was read.

Table 5. Minimum inhibitory concentration (MIC) of chitosan nanogels, Thymol essential oil and Thymol-based chitosan nanoparticles in 3 bacterial genera

\begin{tabular}{|c|c|c|c|c|c|c|c|c|c|c|c|c|c|c|c|c|c|c|}
\hline \multirow{2}{*}{$\begin{array}{c}\text { Bacteria } \\
\text { Sample }\end{array}$} & \multicolumn{6}{|c|}{ Acinetobacter } & \multicolumn{6}{|c|}{ Staphylococcus } & \multicolumn{6}{|c|}{ Pseudomonas } \\
\hline & MIC1 & OD1 & MIC2 & OD2 & MIC3 & OD3 & MIC1 & OD1 & MIC2 & OD2 & MIC3 & OD3 & MIC1 & OD1 & MIC2 & OD2 & MIC3 & OD3 \\
\hline S1 & 128 & 0.221 & 32 & 0.229 & 16 & 0.103 & 256 & 0.339 & 64 & 0.199 & 8 & 0.084 & 64 & 0.273 & 32 & 0.194 & 2 & 0.262 \\
\hline S2 & 64 & 0.301 & 32 & 0.079 & 4 & 0.101 & 128 & 0.267 & 32 & 0.084 & 4 & 0.123 & 128 & 0.199 & 64 & 0.203 & 8 & 0.157 \\
\hline S3 & 256 & 0.358 & 128 & 0.079 & 32 & 0.051 & 64 & 0.271 & 16 & 0.211 & 2 & 0.346 & 256 & 0.115 & 128 & 0.069 & 32 & 0.054 \\
\hline S4 & 512 & 0.128 & 128 & 0.105 & 16 & 0.119 & 128 & 0.244 & 128 & 0.064 & 16 & 0.048 & 256 & 0.271 & 64 & 0.331 & 16 & 0.161 \\
\hline S5 & 128 & 0.312 & 16 & 0.188 & 1 & 0.419 & 256 & 0.302 & 32 & 0.268 & 4 & 0.197 & 128 & 0.244 & 32 & 0.099 & 4 & 0.142 \\
\hline S6 & 512 & 0.329 & 64 & 0.274 & 32 & 0.116 & 128 & 0.292 & 32 & 0.161 & 2 & 0.349 & 256 & 0.346 & 128 & 0.289 & 1 & 0.513 \\
\hline S7 & 128 & 0.394 & 32 & 0.145 & 4 & 0.145 & 256 & 0.284 & 64 & 0.195 & 16 & 0.116 & 128 & 0.328 & 32 & 0.202 & 2 & 0.287 \\
\hline S8 & 256 & 0.312 & 64 & 0.218 & 4 & 0.117 & 256 & 0.291 & 128 & 0.086 & 8 & 0.112 & 64 & 0.386 & 8 & 0.115 & 1 & 0.449 \\
\hline S9 & 128 & 0.347 & 32 & 0.114 & 8 & 0.172 & 128 & 0.401 & 64 & 0.218 & 8 & 0.189 & 128 & 0.396 & 32 & 0.094 & 4 & 0.173 \\
\hline S10 & 256 & 0.301 & 64 & 0.214 & 8 & 0.197 & 64 & 0.315 & 64 & 0.089 & 2 & 0.399 & 256 & 0.318 & 64 & 0.292 & 8 & 0.052 \\
\hline S11 & 256 & 0.412 & 128 & 0.086 & 4 & 0.368 & 256 & 0.215 & 16 & 0.395 & 1 & 0.602 & 32 & 0.486 & 4 & 0.219 & 1 & 0.643 \\
\hline S12 & 512 & 0.159 & 256 & 0.089 & 32 & 0.041 & 512 & 0.286 & 128 & 0.476 & 32 & 0.063 & 128 & 0.239 & 32 & 0.164 & 4 & 0.163 \\
\hline S13 & 128 & 0.326 & 128 & 0.116 & 8 & 0.164 & 128 & 0.326 & 16 & 0.285 & 2 & 0.486 & 256 & 0.213 & 64 & 0.175 & 2 & 0.350 \\
\hline S14 & 64 & 0.315 & 64 & 0.214 & 1 & 0.516 & 128 & 0.112 & 16 & 0.098 & 1 & 0.542 & 128 & 0.315 & 64 & 0.096 & 2 & 0.473 \\
\hline
\end{tabular}




\begin{tabular}{|c|c|c|c|c|c|c|c|c|c|c|c|c|c|c|c|c|c|c|}
\hline S15 & 256 & 0.296 & 64 & 0.215 & 4 & 0.133 & 256 & 0.195 & 32 & 0.102 & 8 & 0.199 & 128 & 0.216 & 32 & 0.118 & 8 & 0.194 \\
\hline S16 & 256 & 0.264 & 32 & 0.112 & 4 & 0.112 & 128 & 0.316 & 64 & 0.064 & 4 & 0.121 & 256 & 0.112 & 64 & 0.114 & 4 & 0.171 \\
\hline S17 & 256 & 0.212 & 32 & 0.127 & 4 & 0.106 & 256 & 0.294 & 64 & 0.118 & 4 & 0.393 & 256 & 0.185 & 32 & 0.186 & 4 & 0.098 \\
\hline S18 & 256 & 0.186 & 64 & 0.202 & 4 & 0.143 & 128 & 0.202 & 32 & 0.102 & 2 & 0.445 & 128 & 0.264 & 64 & 0.126 & 2 & 0.239 \\
\hline S19 & 128 & 0.342 & 32 & 0.132 & 2 & 0.133 & 256 & 0.283 & 16 & 0.231 & 1 & 0.767 & 128 & 0.198 & 64 & 0.102 & 2 & 0.282 \\
\hline S20 & 128 & 0.299 & 64 & 0.143 & 4 & 0.179 & 256 & 0.146 & 128 & 0.098 & 16 & 0.101 & 128 & 0.212 & 32 & 0.186 & 2 & 0.215 \\
\hline S21 & 256 & 0.195 & 64 & 0.096 & 2 & 0.341 & 256 & 0.197 & 32 & 0.111 & 8 & 0.099 & 64 & 0.410 & 32 & 0.186 & 1 & 0.546 \\
\hline S22 & 128 & 0.114 & 32 & 0.235 & 4 & 0.163 & 256 & 0.256 & 64 & 0.146 & 4 & 0.149 & 128 & 0.258 & 64 & 0.199 & 4 & 0.262 \\
\hline S23 & 128 & 0.196 & 32 & 0.113 & 4 & 0.147 & 128 & 0.134 & 32 & 0.187 & 4 & 0.101 & 128 & 0.269 & 32 & 0.178 & 2 & 0.324 \\
\hline S24 & 256 & 0.235 & 64 & 0.102 & 4 & 0.110 & 128 & 0.086 & 32 & 0.043 & 2 & 0.481 & 256 & 0.202 & 64 & 0.120 & 4 & 0.111 \\
\hline S25 & 64 & 0.219 & 4 & 0.343 & 1 & 0.596 & 128 & 0.171 & 64 & 0.086 & 4 & 0.189 & 128 & 0.301 & 32 & 0.62 & 4 & 0.274 \\
\hline S26 & 512 & 0.163 & 128 & 0.169 & 8 & 0.169 & 256 & 0.136 & 64 & 0.071 & 4 & 0.186 & 512 & 0.134 & 64 & 0.098 & 4 & 0.162 \\
\hline S27 & 128 & 0.148 & 32 & 0.123 & 2 & 0.243 & 64 & 0.099 & 8 & 0.158 & 1 & 0.401 & 512 & 0.177 & 128 & 0.147 & 8 & 0.131 \\
\hline ATCC & 512 & 0.365 & 256 & 0.248 & 64 & 0.084 & 512 & 0.389 & 128 & 0.102 & 16 & 0.055 & 512 & 0.201 & 128 & 0.127 & 16 & 0.041 \\
\hline \multicolumn{2}{|c|}{ Abbreviations } & \multicolumn{5}{|c|}{$\begin{array}{l}\text { MIC1= MIC value of thymol essential oil } \\
\text { density }\end{array}$} & & valu & & $\log$ & \multicolumn{6}{|c|}{ MIC3= MIC value of chitosan-thymol nanoparticles } & \multicolumn{2}{|c|}{ OD= Optical } \\
\hline
\end{tabular}

As shown in Figure 4, Staphylococcus bacteria show a higher growth curve at concentrations of 1 to $256 \mu \mathrm{g} / \mathrm{mL}$ against synthesized nanoparticles, indicating that Staphylococcus is more resistant to nanoparticles. Acinetobacter also has a growth curve close to Staphylococcus, although it lacks a cell wall. This indicates the high efficiency of Acinetobacter resistance mechanisms compared to other gram-negative bacteria such as Pseudomonas. Pseudomonas was the most sensitive bacterial strain to the synthesized nanoparticles. On the other hand, Thymol-based chitosan nanoparticles showed the highest inhibitory effect in all three bacterial genera. Concentrations between 256 and $8 \mu \mathrm{g} / \mathrm{mL}$ of Thymolbased chitosan nanoparticles showed the best inhibitory effects in all 3 bacterial genera.

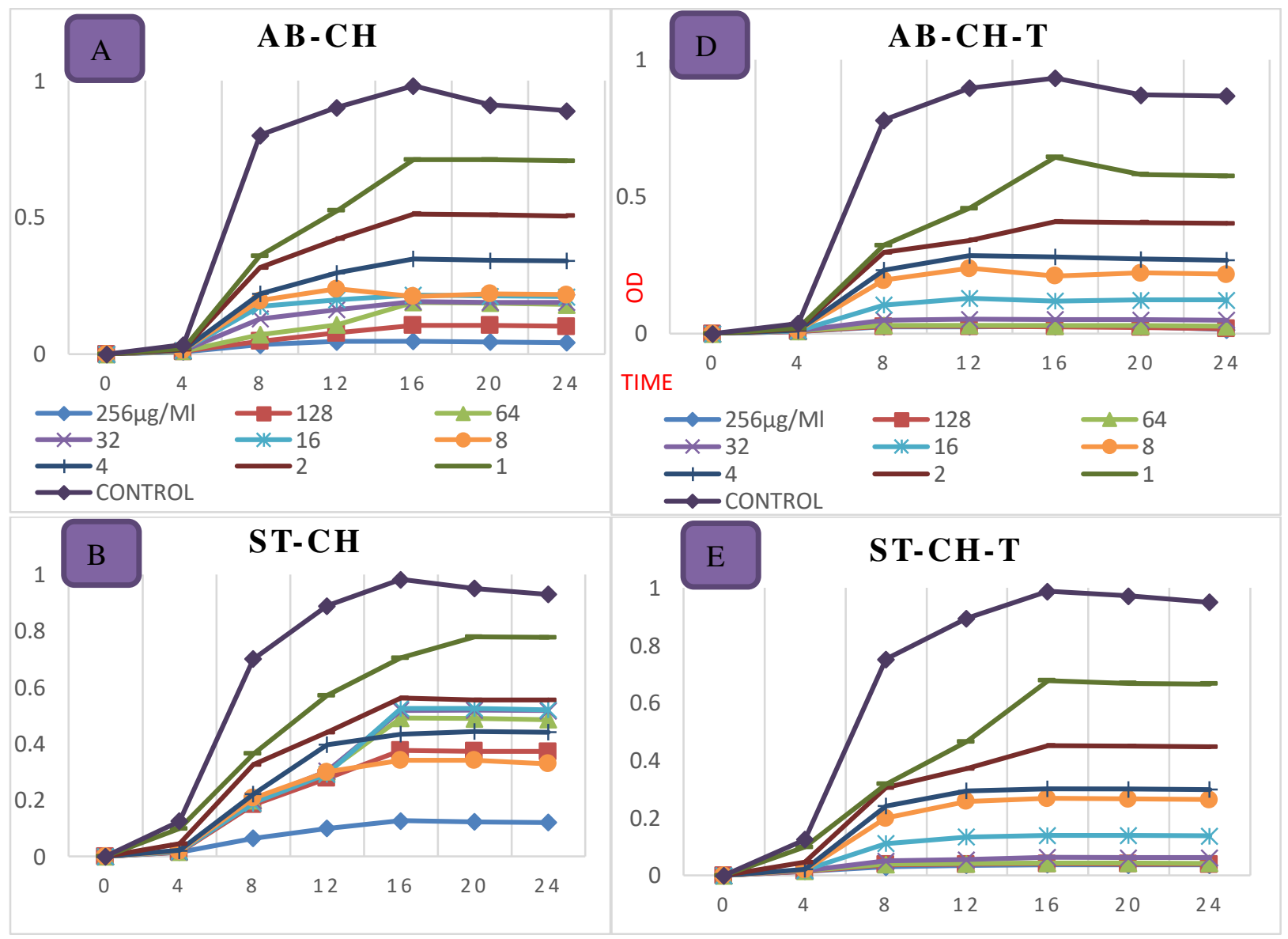




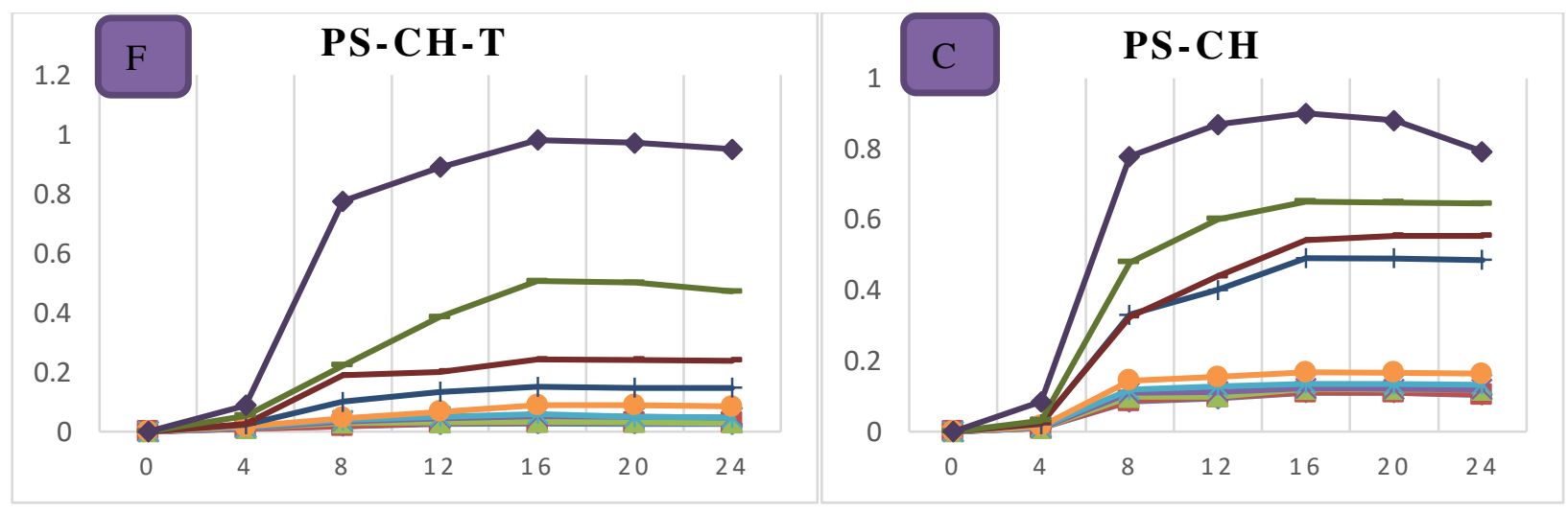

Figure 3. Growth curve of Acinetobacter (A), Staphylococcus (B) and Pseudomonas (C) under the influence of chitosan nanoparticles in 24 hours; Growth curve of Acinetobacter (D), Staphylococcus (E) and Pseudomonas (F) under the influence of Thymol-based chitosan nanoparticles

Then, in order to evaluate the anti-biofilm activity, the subMIC concentration of nanoparticles is used and the expression of $P g a B$ and $O m p A$ genes in all 3 bacterial genera is examined by Quantitative Real-Time PCR and the results of $P g a B$ and $O m p A$ gene expression relative to $16 S r R N A$ reference gene in positive biofilm strains are shown in Graph 2. According to the results, the mean of the highest decrease in $P g a B$ and $O m p A$ gene expression was related to Pseudomonas strain.

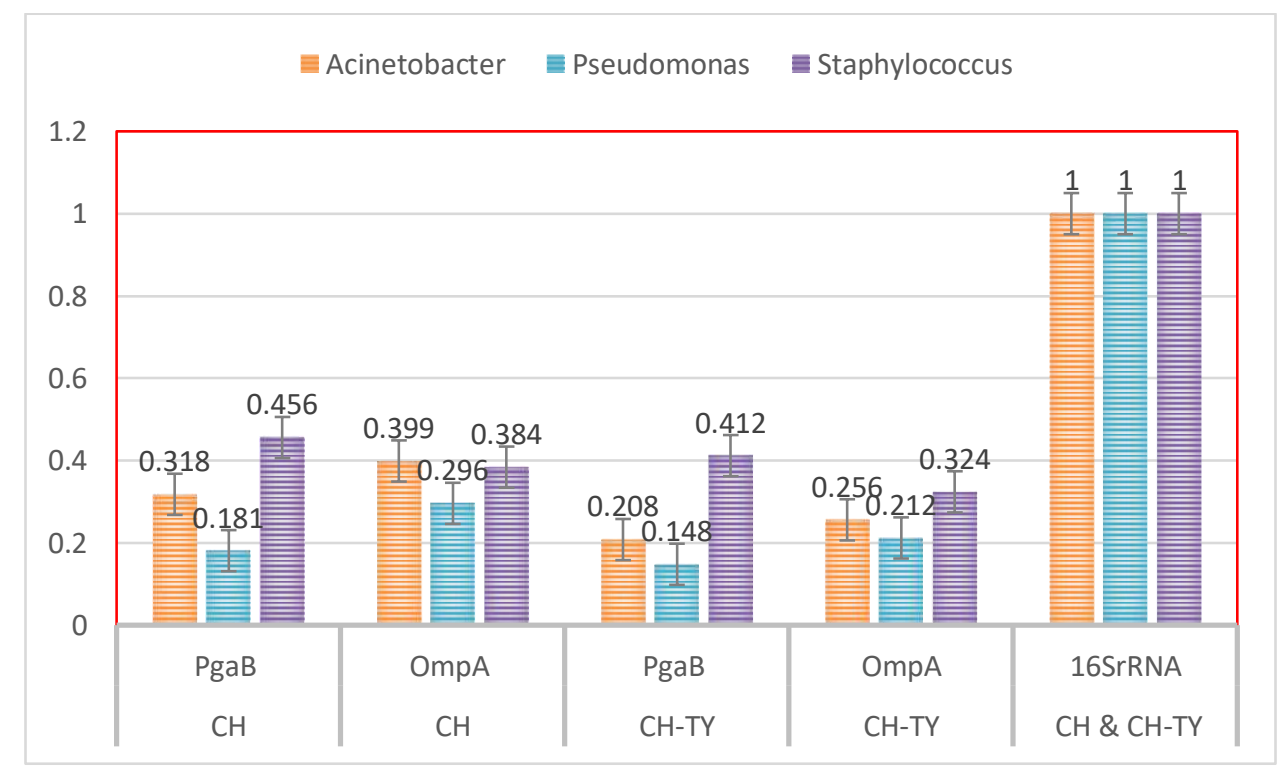

Graph 2. Mean expression of $P g a B$ and $O m p A$ biofilm genes in strains treated with synthesized nanoparticles 
Thymol-based chitosan nanoparticles also showed the greatest inhibitory effect on the expression of biofilm genes in all three bacterial genera; the expression of PgaB gene in Pseudomonas, Acinetobacter, and Staphylococcus bacteria was $0.148,208,0.412$ and the expression of $O m p A$ gene was $0.212,0.256$, and 0.324 , respectively. The results showed that different bacterial strains with different concentrations of MIC, under the influence of chitosan nanogels and Thymol-based chitosan nanoparticles had different expression changes and showed a statistically significant difference compared to the expression of the 16SrRNA gene $(\mathrm{P} \leq 0.05)$. Finally, the antibacterial activity of the synthesized nanoparticles was investigated by the standard method of well diffusion. After culturing Pseudomonas, Acinetobacter, and Staphylococcus separately, four wells were created in the plate containing the culture medium and chitosan nanogels, Thymol-based chitosan nanoparticles, Thymol-free drug, and gentamicin antibiotic were added as positive controls to wells 1 to 4 , respectively.

Table 6. The inhibition zone diameters $(\mathrm{mm})$ of antibacterial compounds in bacteria

\begin{tabular}{|l|c|c|c|}
\hline Compounds & Acinetobacter & Staphylococcus & Pseudomonas \\
\hline Chitosan nanoparticles & 16 & 12 & 21 \\
\hline Thymol-based chitosan nanoparticles & 25 & 18 & 30 \\
\hline Thymol essential oil & 0 & 2 & 3 \\
\hline Gentamicin & 2 & 4 & 5 \\
\hline
\end{tabular}

According to Table 6, the highest growth inhibition zone is related to Thymol-based chitosan nanoparticles, which in Pseudomonas, Acinetobacter, and Staphylococcus bacteria are 30, 25, and $18 \mathrm{~mm}$, respectively. Also, all three bacterial genera were resistant to the antibiotic gentamicin. These results indicate the appropriate antibacterial properties of the synthesized nanoparticles. Finally, the cytotoxicity of chitosan nanogels, free Thymol, and Thymolbased chitosan nanogels were measured at concentrations of 0.125 to $256 \mu \mathrm{g} / \mathrm{mL}$ for HEK-293 cells. According to Figure 7, the results showed that Thymol-based chitosan nanogels had the least inhibitory effect on HEK-293 compared to chitosan nanogels and Thymol-free drug in all samples. 


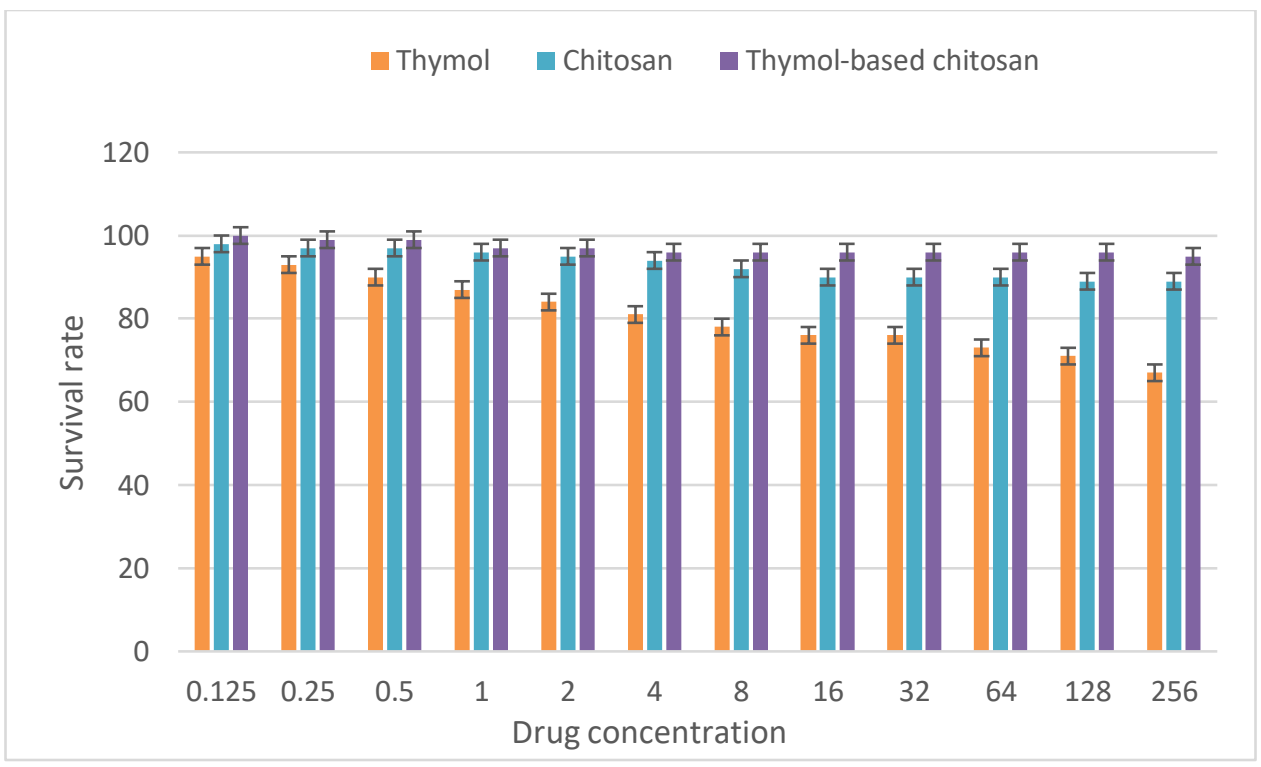

Figure 4. Percentage of HEK-293 cell viability at different drug concentrations

\section{Discussion}

The discovery of antibiotics to treat bacterial infections was one of the most important advances in medical history. Unfortunately, bacteria are very adaptable, and overuse of antibiotics has made many bacteria resistant to antibiotics [23]. With the increase in the range of antibiotic resistance, the density of MDR bacteria in hospitals has increased and has created a new challenge called nosocomial infections [24]. According to the clinical results of this study, the main spectrum of bacteria resistant to various nosocomial infections belonged to the three genera Staphylococcus, Pseudomonas and Acinetobacter. Previous studies have reported an increase in the prevalence of antibiotic resistance in these three genera [25]. On the other hand, the results of the present study showed that $83.3 \%$ of the bacteria are resistant to MDR and have the ability to form biofilms. These results are consistent with the results of previous studies indicates an increase in resistance to various antibiotics due to increased expression of biofilm genes and consequently increased biofilm formation capacity [26]. 66\% of the bacteria studied in this study formed strong biofilms; while other bacteria were Intermediate in terms of biofilm potency, indicating a direct effect of biofilm on antibiotic resistance and high activity of biofilm genes in multidrug-resistant bacteria. According to previous studies, bacteria use various physical, physiological and genetic factors to develop resistance mechanisms, that the highest resistance being related to biofilm and expression of biofilm genes [27]. In this study, it was shown that OmpA and pgaB genes are actively present in MDR bacteria, which increase the ability of biofilms to form in bacteria and contribute to bacterial resistance to various antibiotics. Past studies indicate an important role for the pgaB protein in activating the biofilm response under stress conditions [28]. Many studies have identified $O m p A$ as a factor in antibiotic resistance and biofilm formation [29]. Today, due to the increase in nosocomial infections, the prevalence of MDR bacteria and the slow production of new antibiotics, the need to use natural antibacterial agents is felt more than ever [30]. Therefore, most research is on various strategies to introduce new antibacterial agents or even overcoming bacterial resistance. Therefore, in this study, after preparing chitosan nanogels, encapsulation of Thymol in the structure of chitosan nanogels was performed with the aim of introducing a new antibacterial compound. In the formulation used, 
Thymol, as the main extract of thyme, is a known antibacterial agent (31). While chitosan polymer, due to its positive charge, has a high ability to react with biological membranes (negative charge) and increases the transport capacity of drugs [32]. Chitosan also has different and special properties such as; non-toxic, biodegradable and emulsifying, which is used to convert oily and water-insoluble drugs into soluble form [33]. According to previous studies, chitosan amino groups are responsible for properties such as; Controlled drug release, mucosal adhesion, increased penetration, and bacterial growth inhibitory and inhibitory properties of the efflux pump [34]. By converting chitosan polymer to nanoparticles, its chemical properties can be improved and the transport efficiency of drugs can be increased [35]. Therefore, encapsulation of drugs such as thymol can be promising for new antibacterial compounds. Encapsulation efficiency is an important factor in the synthesis of nanomaterials and various factors such as; Nanoparticle diameters affect capsulation efficiency and consequently drug release ability [36]. The results of our study showed that the capsulation of thymol by chitosan nanogels increases its antibacterial properties. The results of this study are consistent with previous studies that show that the antibacterial properties of thymol are increased at the nano level [37]. In this study, the uniformity of nanoparticles was confirmed by DLS and the size of nanoparticles for chitosan nanogels and Thymol encapsulated in chitosan nanogels were determined to be 258.2 and 179.3 , respectively, while the size of nanoparticles obtained by SEM indicates the appropriate size for chitosan nanogels (72.35) and Thymol encapsulated in chitosan nanogels (82.71). Since DLS determines the hydrodynamic diameter, it consists of nanoparticles and ions or molecules attached to it.

But SEM is the exact method that determines the dry size of nanoparticles. These results, in line with previous studies, indicate the effect of scattering on particle measurement error by DLS [38]. Also, the study of drug release profile showed that drug diffusion at $\mathrm{PH}=7.4$ in 48 hours is $76 \%$. While in the first 9 hours, the highest rate of drug diffusion is seen. The initial diffusion rate can be attributed to the release of surface drugs. As time goes on, the surfaceencapsulated drugs are released more until after 9 hours the Thymol concentration at the surface is minimized and the encapsulated drugs in the underlying layers begin to release. This slows down the release of the drug. Previous studies have introduced drug release control agents in polymer nanoparticles diffusion and biodegradation and reported the rapid release of the drug in the early hours as an "explosion diffusion" [39]. The results of this study in line with previous studies indicate the explosive diffusion of encapsulated Thymol release [40]. Protein stability study showed that temperature as a destructive factor plays an important role in drug preservation [41]. So that with increasing temperature in 60 days interval, the morphological properties of nanoparticles changed and as a result, the efficiency of nanoparticles was decreased. However, according to the results, nanoparticles have a storage capacity of 15 to 30 days with good efficiency. However, time has little effect on the morphology and efficiency of nanoparticles, and thus by storing Thymol-based chitosan nanoparticles at $4{ }^{\circ} \mathrm{C}$, the storage capacity can be increased to more than 60 days. Previous studies have also introduced temperature as a limiting factor in drug storage, so that increasing the temperature causes the fat to lubricate and thus the drug to settle [42]. The main purpose of this study was to evaluate the activity of anti-biofilm and antibacterial activity of thymol-based chitosan nanoparticles. For this purpose, a clinical study was performed on infectious patients of Rasoul Akram Hospital and bacterial density was determined. After initial screening, biofilm formation ability, bacterial density and MDR specificity were evaluated and 3 genera Pseudomonas, Acinetobacter and Staphylococcus were identified as MDR bacteria with positive biofilm with the 
highest density. According to the results of Table 3, Thymol-based chitosan nanoparticles showed the highest growth inhibitory properties against MDR strains of all three bacterial genera. However, Thymol-free drug had the least inhibitory activity. Among the three bacterial genera, Staphylococcus was more resistant than the other two genera. Acinetobacter and Pseudomonas are gram-negative and wallless bacteria, while Staphylococcus has a strong membrane wall, so a higher concentration of the drug is needed for the drug to work effectively. The MBC results were a confirmation of the MIC results. According to studies by Jøraholmen et al. (2020), chitosan has the ability to bind to the outer membrane of gram-negative bacteria as well as penetrate the microbial plasma membrane. This ability serves as strength of chitosan in targeted drug delivery that binds to the surface of bacteria and releases encapsulated drug at the target site [43]. On the other hand, the growth rate of bacteria in 24 hours at different concentrations of chitosan nanogels and Thymol-based chitosan nanoparticles was investigated and the results showed the growth control of microorganisms even at the lowest concentrations of nanoparticles compared to the control. Under normal conditions, the bacterium enters the logarithmic phase after 4 hours and reaches its maximum growth; however, the highest rate of drug release is in the first 9 hours. Therefore, with the highest drug release at the time of maximal bacterial growth, growth control was performed well even at a concentration of $1 \mu \mathrm{g} / \mathrm{L}$ (Figure 5). Based on this study, it can be concluded that the capsulation of Thymol in chitosan nanogels increases its antibacterial properties with targeted drug delivery and drug release control. The expression of OmpA and pgaB biofilm genes in subMIC concentrations of nanoparticles was also measured to evaluate antibiofilm activity. The results showed that the highest reduction in gene expression in all 3 bacteria was related to Thymol-based chitosan nanoparticles, which indicates the interaction of Thymol-based chitosan nanoparticles with transcription factors and thus inhibition of gene expression. Many studies have so far confirmed the relationship between biofilm formation and antibiotic resistance of bacteria [44]. Recently, Parrino et al. (2018) in a study introduced biofilm as the main cause of bacterial virulence [45]. Qi et al. (2016) clearly described the relationship between biofilm formation and antibiotic resistance in bacteria [46]. In various studies, the effect of many natural antibacterial substances and nanoparticles on the control of biofilm formation in bacteria has been investigated [47]. Piri Gharaghieh et al. (2018) introduced silver nanoparticles as particles with biofilm control potential in MDR strains of Acinetobacter baumannii [48]. It seems that Thymol-based nanogels have the ability to bind to the surface of bacterial biofilms and deliver targeted drug due to the specific properties of chitosan. Karygianni et al. (2016) showed a positive correlation between herbal treatments and the rate of elimination of bacterial biofilms in the mouth [49]. In the present study, the antibacterial properties of chitosan nanogels, Thymol-free drug and Thymol-based chitosan nanogels were investigated by well diffusion method and the results showed that Thymol-based chitosan nanogels with the largest diameter of growth inhibition in all three genera of MDR has the best growth inhibition. The results of the present study, consistent with previous studies, reports increased properties of antibacterial agents in combination with chitosan nanoparticles [50].

Kumari et al. (2018) reported the antibacterial activity of Thymol nanoemulsion [51]. Abdelhamid et al. (2017) showed the combined effect of Thymol and silver nanoparticles against Staphylococcus aureus [52]. The last aim of this study was to investigate the non-toxicity of Thymol-based chitosan nanogels on human cells. For this purpose, MTT test was performed and the results showed low or no toxicity of this nanoparticle on HEK-293 cells. These results can be attributed to the amazing properties of chitosan and its non-toxicity. Chitosan can also reduce the toxicity of drug 
agents [53]. Many studies have reported the toxicity-modifying properties of chitosan nanoparticles [54]. Shukla et al. (2015) showed that the cellular damage of iron oxide nanoparticles was significantly reduced after encapsulation in chitosan [55]. The present study also showed that the coating of chitosan nanogels on Thymol reduced cell damage and, as a result, the cytotoxic effect of free Thymol was significantly reduced.

\section{Conclusion}

In summary, chitosan as a drug system based on a polymer acetylated from oral chitin which separates from the hard shell of the shrimp, has promising results for increasing the antibacterial properties of medicinal substances or natural antibacterial substances. Therefore, the encapsulation of Thymol in chitosan nanogels, in addition to increasing the antibacterial and anti-biofilm properties of Thymol, reduces its cytotoxic properties and increases the efficiency of Thymol according to characteristics such as; targeted binding to bacterial levels and drug controlled release. Thymolbased chitosan nanogels also have very strong antibacterial properties against MDR strains such as; Staphylococcus, Acinetobacter, and Pseudomonas, as the challenging bacteria of the century showed. Therefore, the use of Thymolbased chitosan nanogels can be reported as a new strategy in the pharmaceutical industry.

\section{References}

1. Weinberg, S. E., et al. "Control and Management of Multidrug Resistant Acinetobacter baumannii: A review of the evidence and proposal of novel approaches." Infection Prevention in Practice (2020): 100077.

2. Haque, Mainul, et al. "Health care-associated infections-an overview." Infection and drug resistance 11 (2018): 2321.

3. Morosini, María Isabel, and Rafael Cantón. "Changes in bacterial hospital epidemiology." Revista Española de Quimioterapia 31.Suppl 1 (2018): 23.

4. McFee, Robin B. "Nosocomial or hospital-acquired infections: an overview." Disease-a-Month 55.7 (2009): 422.

5. Nouri, Fatemeh, et al. "Prevalence of common nosocomial infections and evaluation of antibiotic resistance patterns in patients with secondary infections in Hamadan, Iran." Infection and Drug Resistance 13 (2020): 2365.

6. Fair, Richard J., and Yitzhak Tor. "Antibiotics and bacterial resistance in the 21st century." Perspectives in medicinal chemistry 6 (2014): PMC-S14459.

7. Davies, Julian, and Dorothy Davies. "Origins and evolution of antibiotic resistance." Microbiology and molecular biology reviews 74.3 (2010): 417-433.

8. Verderosa, Anthony Daniel, Makrina Totsika, and Kathryn E. Fairfull-Smith. "Bacterial biofilm eradication agents: a current review." Frontiers in Chemistry 7 (2019): 824.

9. Colquhoun, Jennifer M., and Philip N. Rather. "Insights into Mechanisms of Biofilm Formation in Acinetobacter baumannii and Implications for Uropathogenesis." Frontiers in Cellular and Infection Microbiology 10 (2020): 253.

10. Jang, In-Ae, Jisun Kim, and Woojun Park. "Endogenous hydrogen peroxide increases biofilm formation by inducing exopolysaccharide production in Acinetobacter oleivorans DR1." Scientific reports 6.1 (2016): 1-12. 
11. Little, Dustin J., et al. "The structure-and metal-dependent activity of Escherichia coli PgaB provides insight into the partial de-N-acetylation of poly- $\beta-1,6-\mathrm{N}$-acetyl-D-glucosamine." Journal of Biological Chemistry 287.37 (2012): 31126-31137.

12. Abadi, Amin Talebi Bezmin, et al. "World Health Organization Report: Current Crisis of Antibiotic Resistance." BioNanoScience 9.4 (2019): 778-788.

13. Cheesman, Matthew J., et al. "Developing new antimicrobial therapies: are synergistic combinations of plant extracts/compounds with conventional antibiotics the solution?" Pharmacognosy reviews 11.22 (2017): 57.

14. Anand, Uttpal, et al. "A comprehensive review on medicinal plants as antimicrobial therapeutics: potential avenues of biocompatible drug discovery." Metabolites 9.11 (2019): 258.

15. Salehi, Bahare, et al. "Thymol, thyme, and other plant sources: Health and potential uses." Phytotherapy Research 32.9 (2018): 1688-1706.

16. Yuan, Wenqian, and Hyun-Gyun Yuk. "Effects of sublethal thymol, carvacrol, and trans-cinnamaldehyde adaptation on virulence properties of Escherichia coli O157: H7." Applied and Environmental Microbiology 85.14 (2019): e00271-19.

17. Chouhan, Sonam, Kanika Sharma, and Sanjay Guleria. "Antimicrobial activity of some essential oils—present status and future perspectives." Medicines 4.3 (2017): 58.

18. Fathi-Achachelouei, Milad, et al. "Use of nanoparticles in tissue engineering and regenerative medicine." Frontiers in bioengineering and biotechnology 7 (2019): 113.

19. Jeevanandam, Jaison, et al. "Review on nanoparticles and nanostructured materials: history, sources, toxicity and regulations." Beilstein journal of nanotechnology 9.1 (2018): 1050-1074.

20. Lombardo, Domenico, Mikhail A. Kiselev, and Maria Teresa Caccamo. "Smart nanoparticles for drug delivery application: development of versatile nanocarrier platforms in biotechnology and nanomedicine." Journal of Nanomaterials 2019 (2019).

21. Kravanja, Gregor, et al. "Chitosan-based (Nano) materials for novel biomedical applications." Molecules 24.10 (2019): 1960.

22. Mariana, N. S., et al. "Evaluation of modified Congo red agar for detection of biofilm produced by clinical isolates of methicillinresistance Staphylococcus aureus." African Journal of Microbiology Research 3.6 (2009): 330-338.

23. Lewis, Kim. "Platforms for antibiotic discovery." Nature reviews Drug discovery 12.5 (2013): 371-387.

24. Ventola, C. Lee. "The antibiotic resistance crisis: part 1: causes and threats." Pharmacy and therapeutics 40.4 (2015): 277.

25. Pachori, Preeti, Ragini Gothalwal, and Puneet Gandhi. "Emergence of antibiotic resistance Pseudomonas aeruginosa in intensive care unit; a critical review." Genes \& diseases 6.2 (2019): 109-119.

26. Kamali, Esmat, et al. "Evaluation of antimicrobial resistance, biofilm forming potential, and the presence of biofilm-related genes among clinical isolates of Pseudomonas aeruginosa." BMC Research Notes 13.1 (2020): 27.

27. Vestby, Lene K., et al. "Bacterial Biofilm and its Role in the Pathogenesis of Disease." Antibiotics 9.2 (2020): 59.

28. Boehm, Alex, et al. "Second messenger signalling governs Escherichia coli biofilm induction upon ribosomal stress." Molecular microbiology 72.6 (2009): 1500-1516. 
29. Longo, Francesca, Claudia Vuotto, and Gianfranco Donelli. "Biofilm formation in Acinetobacter baumannii." New Microbiol 37.2 (2014): 119-127.

30. Rochford, Connor, et al. "Global governance of antimicrobial resistance." The Lancet 391.10134 (2018): 19761978.

31. Marchese, Anna, et al. "Antibacterial and antifungal activities of thymol: A brief review of the literature." Food chemistry 210 (2016): 402-414.

32. Garg, Unnati, et al. "Current advances in chitosan nanoparticles based drug delivery and targeting." Advanced pharmaceutical bulletin 9.2 (2019): 195.

33. Islam, Mohammad Ariful, et al. "Design and application of chitosan microspheres as oral and nasal vaccine carriers: an updated review." International journal of nanomedicine 7 (2012): 6077.

34. Muxika, A., et al. "Chitosan as a bioactive polymer: Processing, properties and applications." International Journal of Biological Macromolecules 105 (2017): 1358-1368.

35. Wu, Yuanbing, et al. "Chitosan-Based Drug Delivery System: Applications in Fish Biotechnology." Polymers 12.5 (2020): 1177.

36. Chenthamara, Dhrisya, et al. "Therapeutic efficacy of nanoparticles and routes of administration." Biomaterials Research 23.1 (2019): 1-29.

37. Wattanasatcha, Anna, Sirirat Rengpipat, and Supason Wanichwecharungruang. "Thymol nanospheres as an effective anti-bacterial agent." International Journal of Pharmaceutics 434.1-2 (2012): 360-365.

38. Malm, Alexander V., and Jason CW Corbett. "Improved dynamic light scattering using an adaptive and statistically driven time resolved treatment of correlation data." Scientific reports 9.1 (2019): 1-11.

39. Lee, Jinhyun Hannah, and Yoon Yeo. "Controlled drug release from pharmaceutical nanocarriers." Chemical engineering science 125 (2015): 75-84.

40. Zhang, Yibo, et al. "Encapsulation of thymol in biodegradable nanofiber via coaxial eletrospinning and applications in fruit preservation." Journal of agricultural and food chemistry 67.6 (2019): 1736-1741.

41. Jol, Simon, et al. "Issues in time and temperature abuse of refrigerated foods." Foodsafety magazine (2005).

42. Sykes, Claire. "Time-and temperature-controlled transport: supply chain challenges and solutions." Pharmacy and Therapeutics 43.3 (2018): 154.

43. Jøraholmen, May Wenche, et al. "The Antimicrobial Properties of Chitosan Can be Tailored by Formulation." Marine Drugs 18.2 (2020): 96.

44. Dumaru, Rabina, Ratna Baral, and Lok Bahadur Shrestha. "Study of biofilm formation and antibiotic resistance pattern of gram-negative Bacilli among the clinical isolates at BPKIHS, Dharan." BMC Research Notes 12.1 (2019): 38.

45. Parrino, Barbara, et al. "Bacterial biofilm inhibition in the development of effective anti-virulence strategy." The Open Medicinal Chemistry Journal 12 (2018): 84.

46. Qi, Lihua, et al. "Relationship between antibiotic resistance, biofilm formation, and biofilm-specific resistance in Acinetobacter baumannii." Frontiers in microbiology 7 (2016): 483. 
47. Vallet-Regí, María, Blanca González, and Isabel Izquierdo-Barba. "Nanomaterials as promising alternative in the infection treatment." International journal of molecular sciences 20.15 (2019): 3806.

48. Piri Gharaghie, Tohid, and Seyed Ataollah Sadat Shandiz. "The Inhibitory Effects of Silver Nanoparticles on Bap Gene Expression in Antibiotic-Resistant Acientobacter bumanni Isolates using Real-Time PCR." scientific journal of ilam university of medical sciences 26.4 (2018): 175-185.

49. Karygianni, Lamprini, et al. "Natural antimicrobials and oral microorganisms: a systematic review on herbal interventions for the eradication of multispecies oral biofilms." Frontiers in Microbiology 6 (2016): 1529.

50. Atay, Hüsnügül Yilmaz. "Antibacterial Activity of Chitosan-Based Systems." Functional Chitosan. Springer, Singapore, 2019. 457-489.

51. Kumari, Sarita, et al. "Thymol nanoemulsion exhibits potential antibacterial activity against bacterial pustule disease and growth promotory effect on soybean." Scientific reports 8.1 (2018): 1-12.

52. Abdelhamid, Sarah M., and Lobna S. El-Hosseiny. "Combined efficacy of thymol and silver nanoparticles against Staphylococcus aureus." African Journal of Microbiology Research 11.11 (2017): 450-457.

53. Rizeq, Balsam R., et al. "Synthesis, bioapplications, and toxicity evaluation of chitosan-based nanoparticles." International journal of molecular sciences 20.22 (2019): 5776.

54. Sedyakina, Natalia, et al. "Modulation of Entrapment Efficiency and In Vitro Release Properties of BSA-Loaded Chitosan Microparticles Cross-Linked with Citric Acid as a Potential Protein-Drug Delivery System." Materials 13.8 (2020): 1989.

55. Shukla, Sudeep, et al. "In vitro toxicity assessment of chitosan oligosaccharide coated iron oxide nanoparticles." Toxicology reports 2 (2015): 27-39. 


\section{Figures}

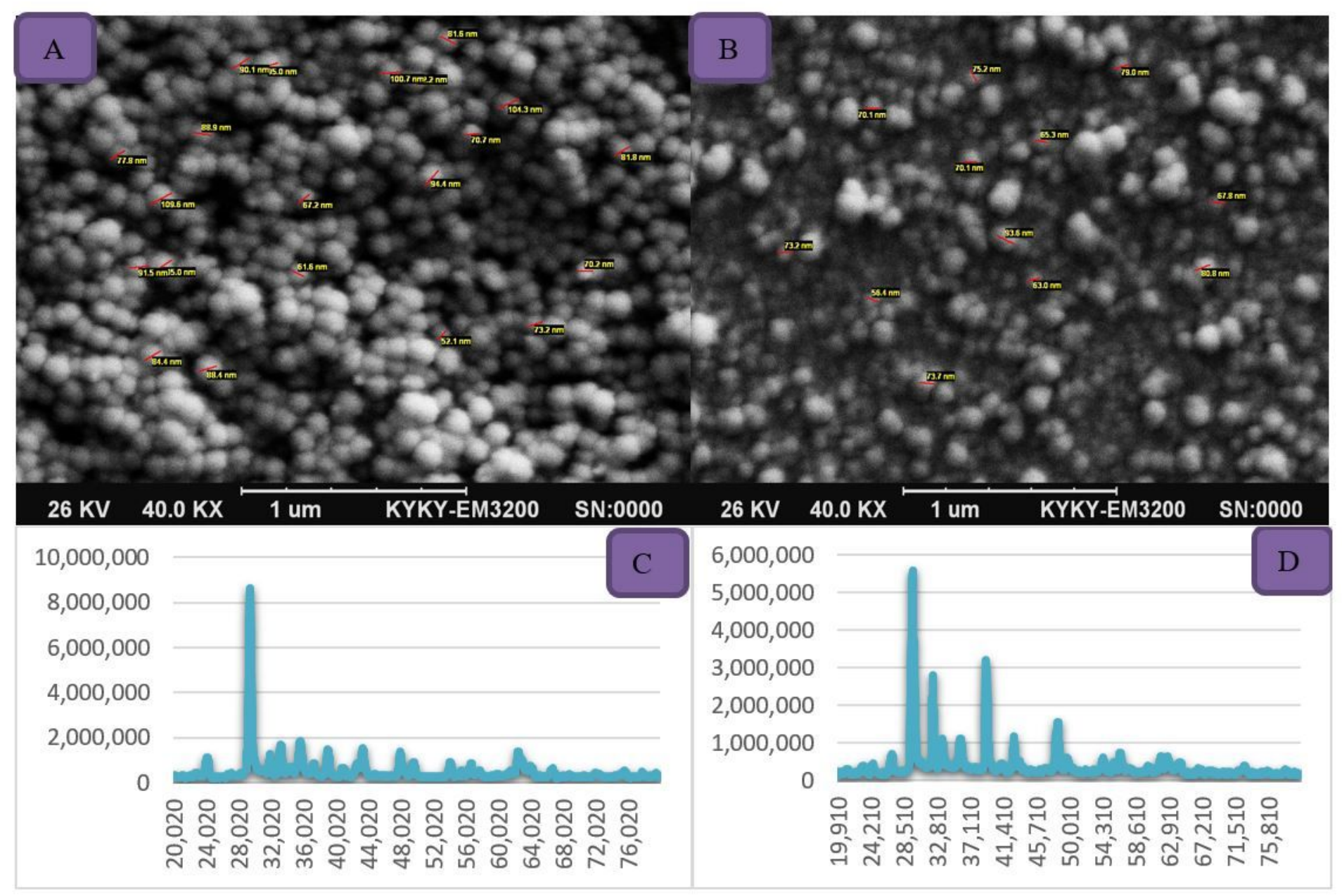

\section{Figure 1}

A) SEM image of chitosan; B) SEM image of chitosan nanoparticles containing Thymol; C) XRD diagram of chitosan nanoparticles; D) XRD diagram of chitosan nanoparticles containing Thymol 

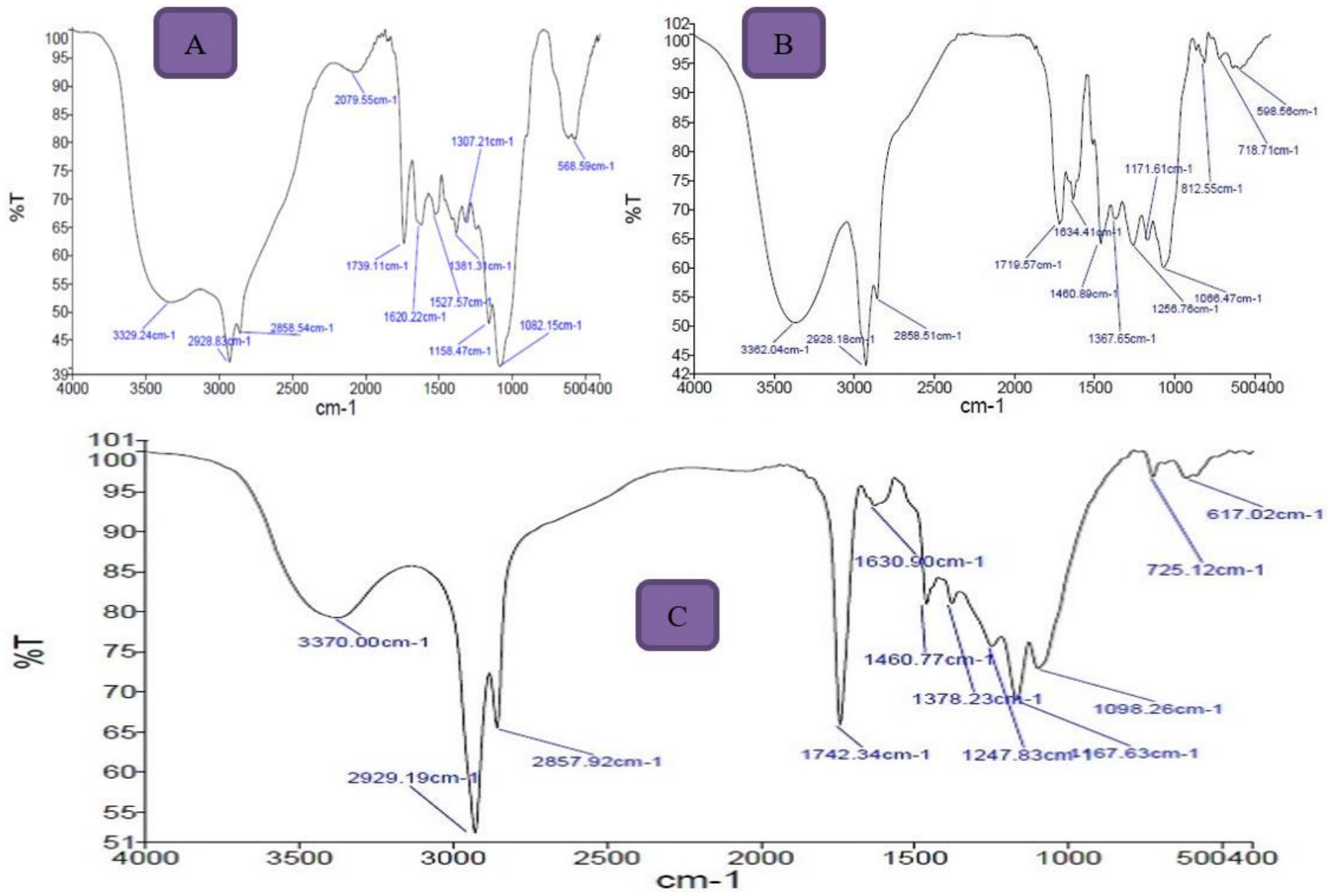

Figure 2

A) FTIR spectrum of chitosan; B) FTIR spectrum of Thymol free drug; c) FTIR spectrum of chitosan nanoparticles containing Thymol 

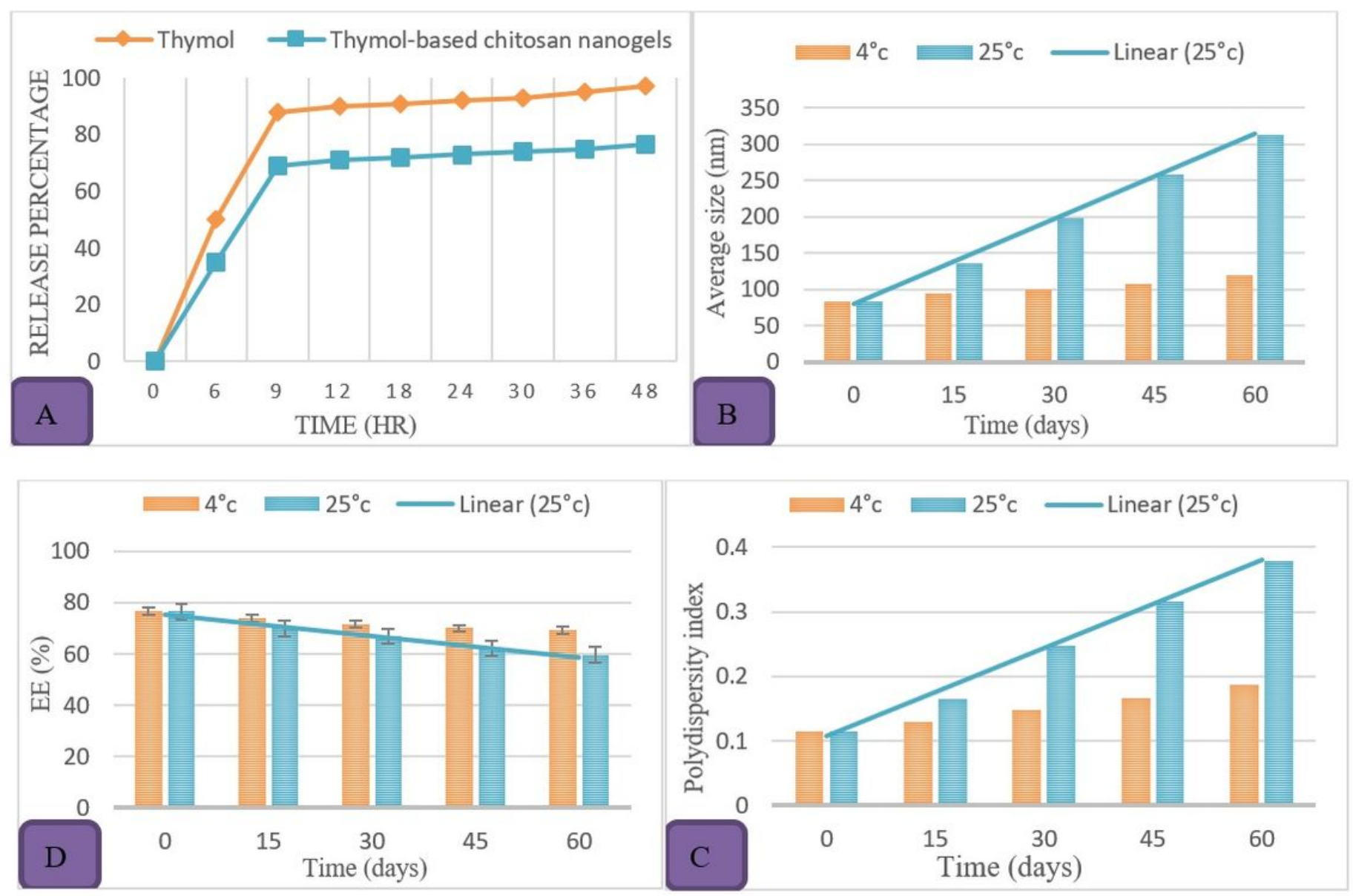

\section{Figure 3}

(A) Evaluation of drug release test; (B) the effect of time and storage temperature on mean size; (C) PDI index; (D) encapsulation efficiency of Thymol-based chitosan nanoparticles. 

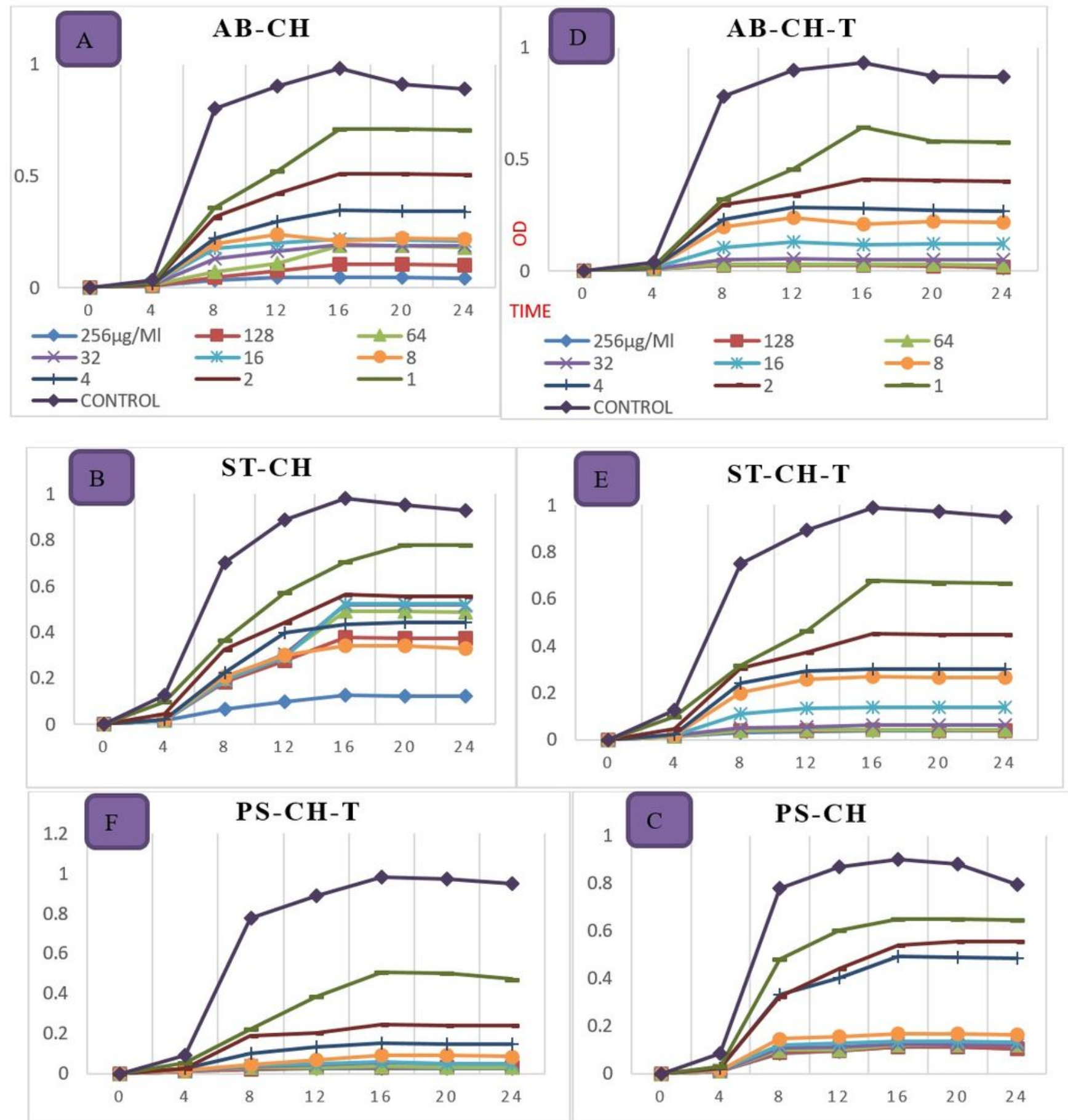

\section{Figure 4}

Growth curve of Acinetobacter (A), Staphylococcus (B) and Pseudomonas (C) under the influence of chitosan nanoparticles in 24 hours; Growth curve of Acinetobacter (D), Staphylococcus (E) and Pseudomonas $(F)$ under the influence of Thymol-based chitosan nanoparticles 


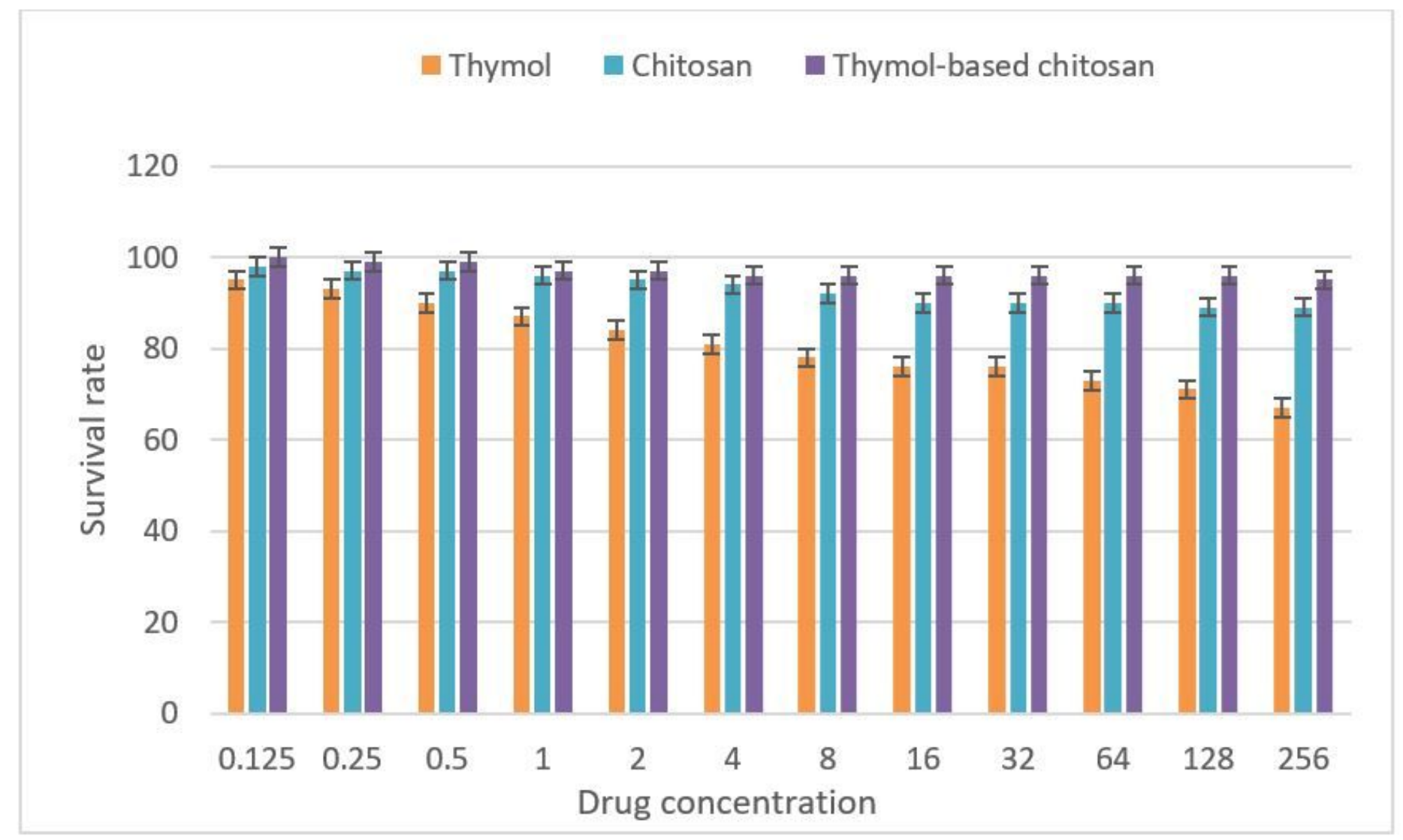

Figure 5

Percentage of HEK-293 cell viability at different drug concentrations

\section{Supplementary Files}

This is a list of supplementary files associated with this preprint. Click to download.

- Graph1.jpg

- Graph2.jpg 